\title{
Regioselective Reductive Opening of Benzylidene Acetals with \\ Dichlorophenylborane/Triethylsilane: Previously Unreported Side- \\ Reactions and How to Prevent them.
}

\section{Supporting Information}

\author{
Jérome Hénault, ${ }^{1}$ Pauline Quellier, ${ }^{1}$ Maxime Mock-Joubert, ${ }^{1}$ Christine Le Narvor, ${ }^{1}$ Aurélien Alix ${ }^{1}$ \\ and David Bonnaffé ${ }^{\star, 1}$ \\ ${ }^{1}$ Université Paris-Saclay, CNRS, Institut de chimie moléculaire et des matériaux d'Orsay, 91405, Orsay, France, \\ david.bonnaffe@universite-paris-saclay.fr
}

\section{Table of contents}

1. $\mathrm{PhBCl}_{2}{ }^{1} \mathrm{H}$ NMR titration and $\mathrm{NMR}$ data for $\mathrm{PhBCl}_{2} / \mathrm{PhSiMe}_{3}$ mixture S2

1.1. $\mathrm{PhBCl}_{2}{ }^{1} \mathrm{H}$ NMR titration on hydrolyzed aliquot $\mathrm{S} 2$

1.2. ${ }^{1} \mathrm{H},{ }^{13} \mathrm{C}-\left\{{ }^{1} \mathrm{H}\right\},{ }^{13} \mathrm{C}-\mathrm{DEPT}-135,{ }^{1} \mathrm{H}-{ }^{13} \mathrm{C} \mathrm{HSQC}$ and ${ }^{11} \mathrm{~B}$ NMR data for $\mathrm{PhBCl}_{2} / \mathrm{PhSiMe}_{3}$ mixture S4

2. ${ }^{1} \mathrm{H}$ and ${ }^{13} \mathrm{C}$ NMR data for compounds $3 \mathrm{a}, 4 \mathrm{a}, 5 \mathrm{a}, 5 \mathrm{~b}, 6 \mathrm{a}, 6 \mathrm{~b}, 8 \mathrm{a}$ and $8 \mathrm{~b} \quad S 7$

2.1. Allyl 2-azido-3,4-di-O-benzyl-2-deoxy- $\alpha$-D-glucopyranoside (3a)

2.2. Allyl (2-azido-3,4-di-O-benzyl-2-deoxy- $\alpha$-D-glucopyranosyl)-(1 $\rightarrow 4)$-(methyl 2-O-acetyl-3-O-benzyl-2deoxy- $\alpha$-L-idopyranuronyl)-(1 $\rightarrow 4)-6-O$-acetyl-2-azido-3-O-benzyl-2-deoxy- $\alpha$-D-glucopyranoside (4a)

2.3. 3-hydroxypropyl 2-azido-3,4-di-O-benzyl-2-deoxy- $\alpha$-D-glucopyranoside (5a) and 2-hydroxypropyl 2azido-3,4-di-O-benzyl-2-deoxy- $\alpha$-D-glucopyranoside (5b).

2.4. 3-hydroxypropyl (2-azido-3,4-di-O-benzyl-2-deoxy- $\alpha$-D-glucopyranosyl)-(1->4)-(methyl 2-O-acetyl-3-Obenzyl-2-deoxy- $\alpha$-L-idopyranuronyl)-(1->4)-6-O-acetyl-2-azido-3-O-benzyl-2-deoxy- $\alpha$-D-glucopyranoside (6a)

2.5. 3-hydroxypropyl (2-azido-3,4-di-O-benzyl-2-deoxy- $\alpha$-D-glucopyranosyl)-(1->4)-(methyl 2-O-acetyl-3-Obenzyl-2-deoxy- $\alpha$-L-idopyranuronyl)-(1->4)-6-O-acetyl-2-azido-3-O-benzyl-2-deoxy- $\alpha$-D-glucopyranoside (6b)

2.6. 3-hydroxypropyl 2-azido-3,4,6-tri-O-benzyl-2-deoxy- $\alpha$-D-glucopyranoside (8a)

2.7. 2-hydroxypropyl 2-azido-3,4,6-tri-O-benzyl-2-deoxy- $\alpha$-D-glucopyranoside (8b)

4. ${ }^{1} \mathrm{H},{ }^{13} \mathrm{C}-\{1 \mathrm{H}\}, \operatorname{COSY},{ }^{1} \mathrm{H}-{ }^{13} \mathrm{C}$ HSQC, ${ }^{1} \mathrm{H}-{ }^{13} \mathrm{C}$ HMBC and HRMS data for compound 10 


\section{1. $\mathrm{PhBCl}_{2}{ }^{1} \mathrm{H}$ NMR titration and $\mathrm{NMR}$ data for $\mathrm{PhBCl}_{2} / \mathrm{PhSiMe}_{3}$ mixture}

\section{1. $\mathrm{PhBCl}_{2}{ }^{7} \mathrm{H}$ NMR titration on hydrolyzed aliquot}

As described in the Result and Discussion" section: "the conventional distillation apparatus we used to purify $\mathrm{PhBCl}_{2}$ did not allowed us to separate the Lewis acid from unreacted $\mathrm{PhSiMe}$. We thus obtained $\mathrm{PhBCl}_{2} / \mathrm{PhSiMe}_{3}$ mixtures, in reproducible $\approx 65 / 35$ molar ratio, that can be further used without additional purification for reductive opening reactions and can be stored for at least five years in sealed ampules." Even if the $\mathrm{PhBCl}_{2} / \mathrm{PhSiMe}_{3}$ ratio is reproductible, we always check it for each new batch prepared by NMR titration. Although ${ }^{1} \mathrm{H},{ }^{13} \mathrm{C}-\left\{{ }^{1} \mathrm{H}\right\}$ and ${ }^{11} \mathrm{~B}$ NMR spectra of the $\mathrm{PhBCl}_{2} / \mathrm{PhSiMe}_{3}$ mixture can be recorded (see below), strict anhydrous conditions must be maintained to avoid partial hydrolysis. We found more convenient to determine the $\mathrm{PhBCl}_{2} / \mathrm{PhSiMe}_{3}$ ratio on a fully hydrolyzed aliquot. To this aim, $20 \mathrm{mg}$ of the $\mathrm{PhBCl}_{2} / \mathrm{PhSiMe}_{3}$ mixture were dissolved in $500 \mu \mathrm{L}$ of $\mathrm{CDCl}_{3}$ in an NMR tube and $100 \mu \mathrm{L}$ of $\mathrm{D}_{2} \mathrm{O}$ were added. The NMR tube was thoroughly shaken, the mixture was decanted and a ${ }^{1} \mathrm{H}$ NMR spectra was recorded at $400 \mathrm{MHz}$. After Fourier Transform, phase and baseline corrections, the integration of the aromatic (Int $\mathrm{Arom}_{\text {) }}$ and $\mathrm{SiMe}_{3}$ (Int $\mathrm{TMS}_{\mathrm{T}}$ ) protons were determined, from which the molar \% compositions were easily derived:

$$
n \%_{\text {PhBCl }_{2}}=\frac{\text { Int }_{\text {Arom }}-5 / 9 \text { Int }_{T M S}}{\text { Int }_{\text {Arom }}} \text { and } n \%_{\text {PhTMS }}=\frac{5 \text { Int }_{T M S}}{9 \text { Int }_{\text {Arom }}}
$$

In the present case (batch 1): $n \%_{P_{P B C l}}=65 \%$ and $n \%_{P h T M S}=35 \%$

Corresponding to the following mass \% composition: $m \%_{\text {PhBCl }_{2}}=66 \%$ and $m \%_{\text {PhTMS }}=34 \%$

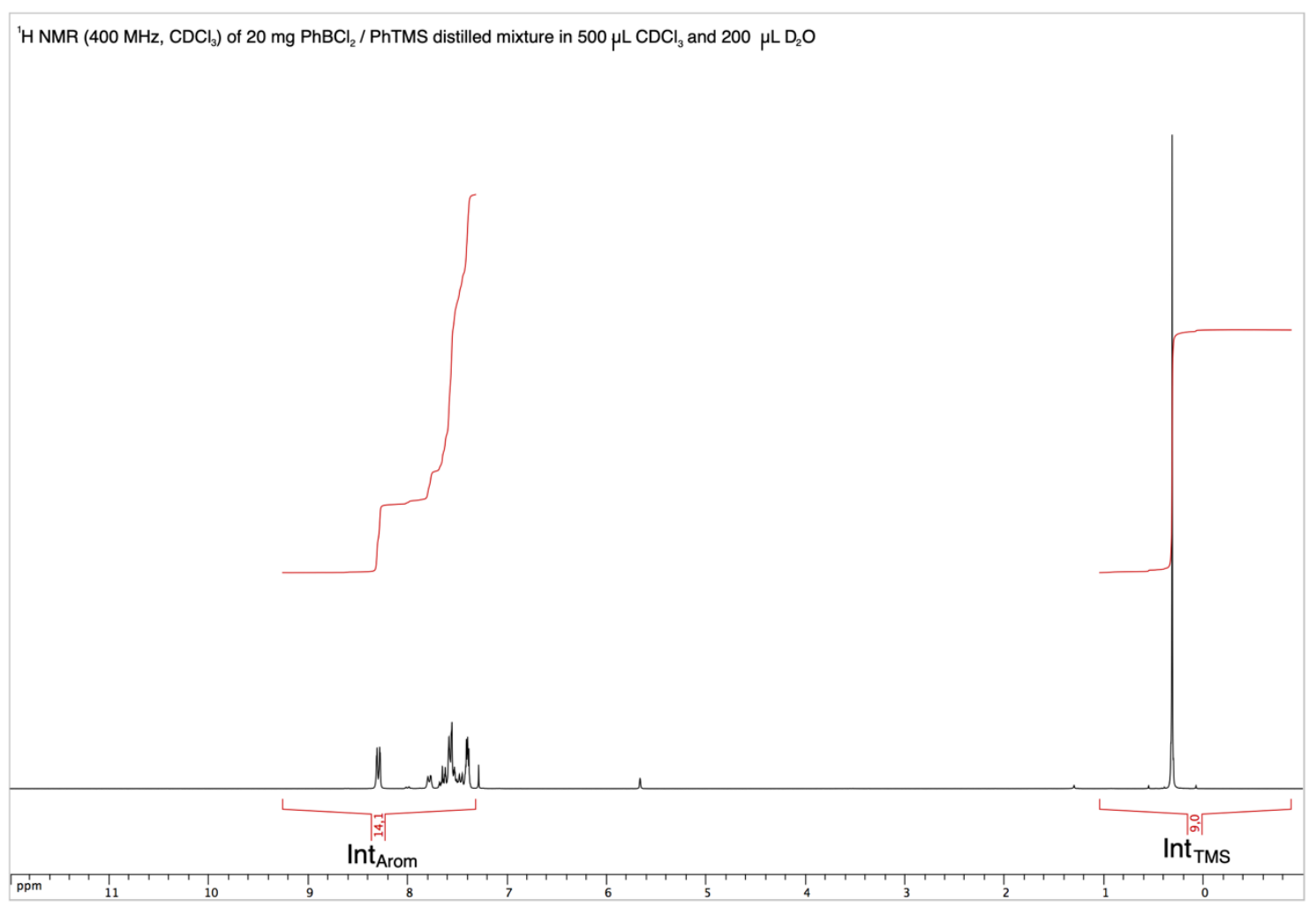


A closer look at the aromatic region of the ${ }^{1} \mathrm{H}$ NMR spectrum reveal that the formed phenyl boronic acid exists as a mixture of trimer, dimer and monomer, whose aromatic protons resonates at $\delta=8.30,8.01$ and $7.78 \mathrm{ppm}$ respectively. ${ }^{1}$ One may thus wonder whether part of the more hydrophilic dimer and monomer could have been extracted in the $\mathrm{D}_{2} \mathrm{O}$ layer. To rule out this hypothesis, we decided to perform a quantitative titration by adding hexamethylbenzene as internal standard. To this aim, $20 \mathrm{mg}$ of the $\mathrm{PhBCl}_{2} / \mathrm{PhSiMe}_{3}$ mixture were added to $500 \mu \mathrm{L}$ of a 5 $\mathrm{mg} \cdot \mathrm{mL}^{-1}(31 \mathrm{mM})$ solution of hexamethylbenzene in $\mathrm{CDCl}_{3}$ in an NMR tube, followed by $100 \mu \mathrm{L}$ of $\mathrm{D}_{2} \mathrm{O}$. The NMR tube was thoroughly shaken, the mixture was decanted and a ${ }^{1} \mathrm{H}$ NMR spectra was recorded at $400 \mathrm{MHz}$ and is displayed below.

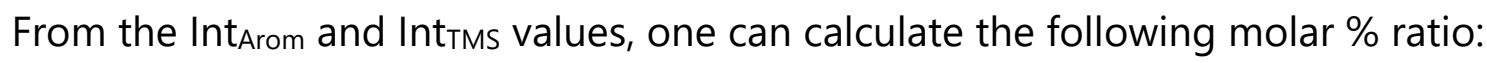

$$
n \%_{\text {PhBCl }_{2}}=64 \% \text { and } n \%_{\text {PhTMS }}=36 \%
$$

From the integration of the signals of the ortho protons of the phenylboronic derivatives (10.6) and of the hexamethylbenzene (18.0) and TMS (26.4) ones, one can calculate $\mathrm{PhB}(\mathrm{OH})_{2}$ derivatives and PhTMS concentrations of 160 and $90 \mathrm{mM}$ respectively. This corresponds to a $64 / 36$ molar ratio, indicating that no phenylboronic derivatives have been extracted in the $\mathrm{D}_{2} \mathrm{O}$ layer.

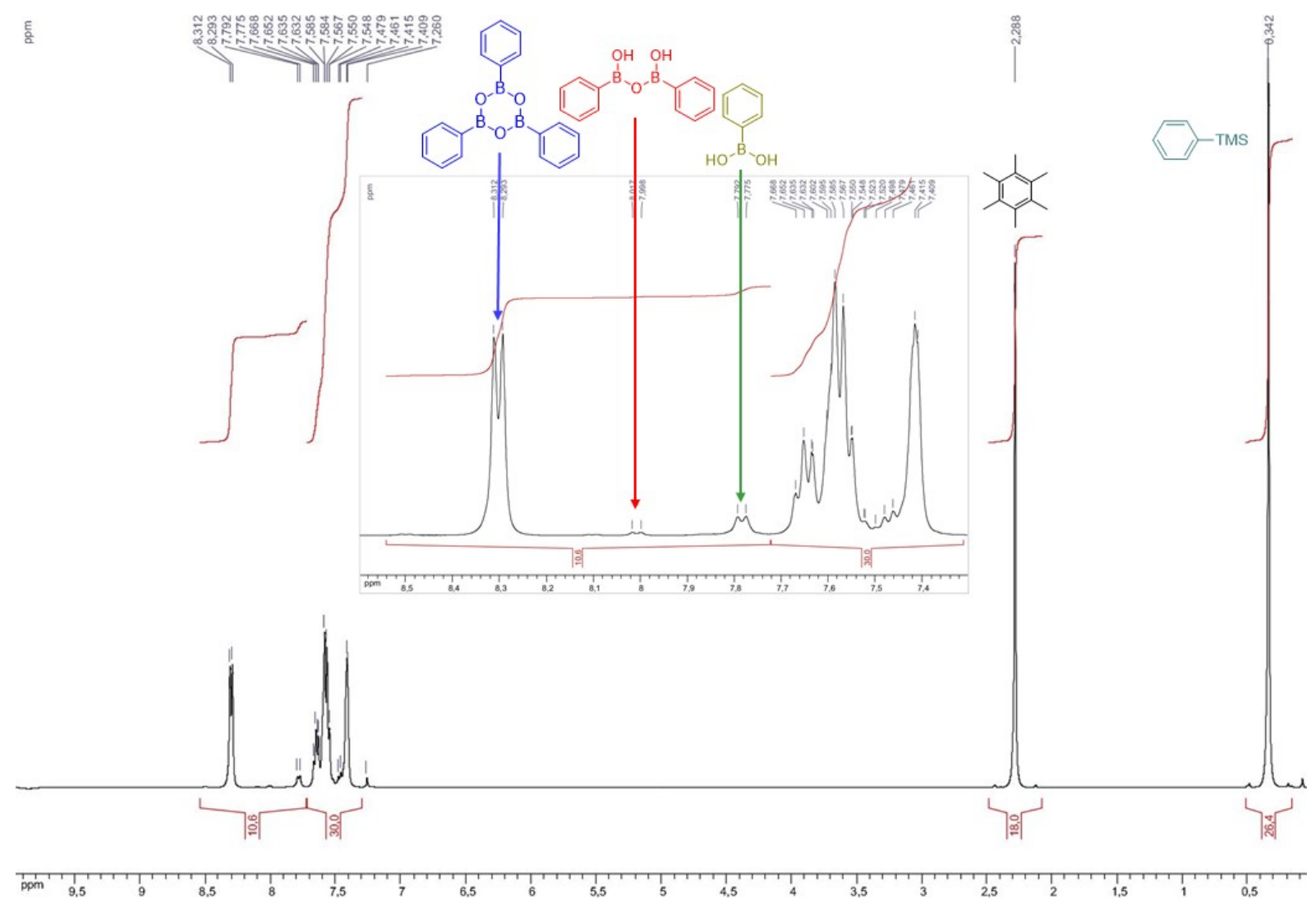


1.2. ${ }^{1} \mathrm{H},{ }^{13} \mathrm{C}-\left\{{ }^{1} \mathrm{H}\right\}$ and ${ }^{11} \mathrm{~B}$ NMR data for $\mathrm{PhBCl}_{2} / \mathrm{PhSiMe}_{3}$ mixture ${ }^{1} \mathrm{H} \mathrm{NMR}_{1} \mathrm{CDCl}_{3}, 300 \mathrm{MHz}$

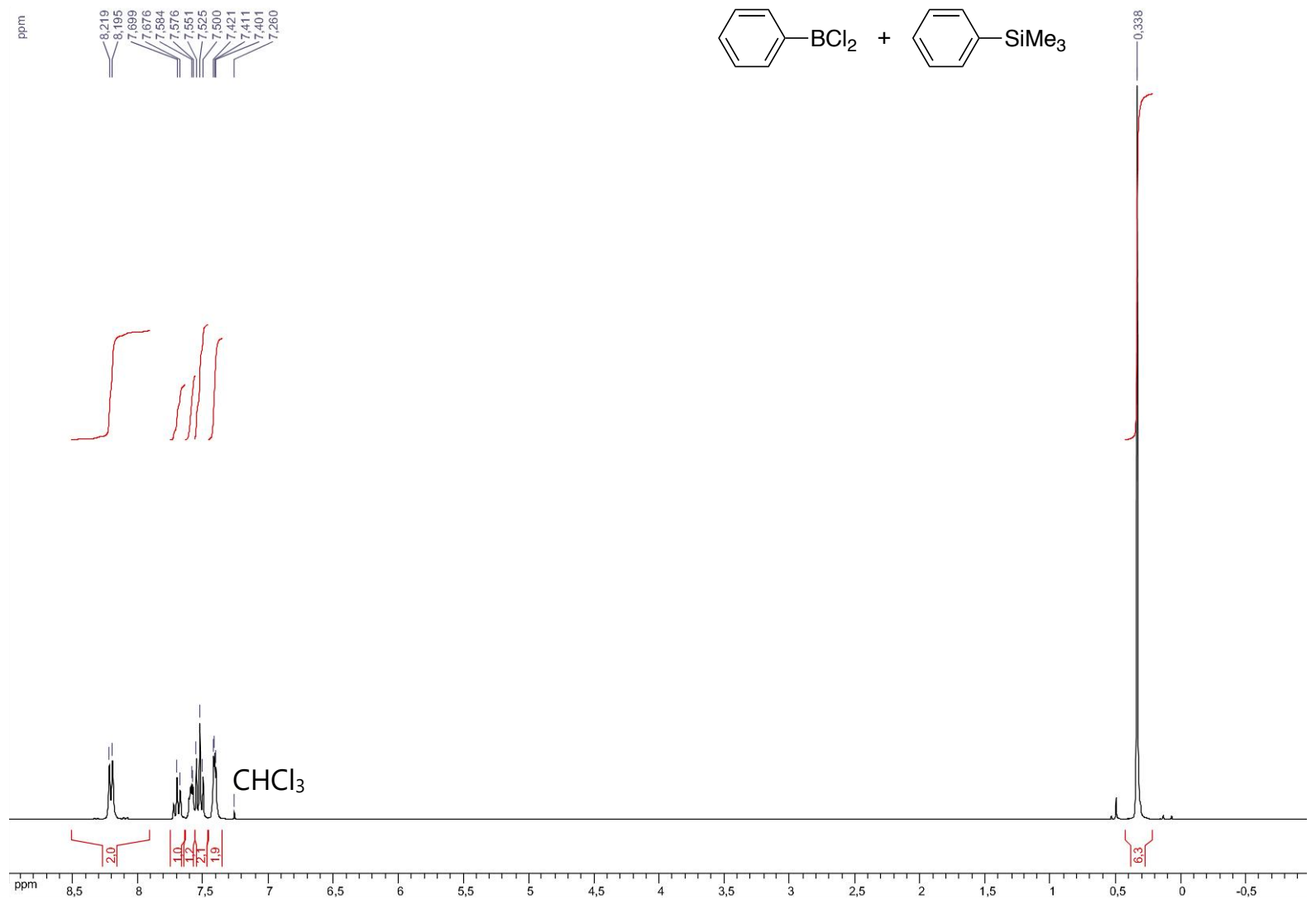

${ }^{13} \mathrm{C}-\left\{{ }^{1} \mathrm{H}\right\} \mathrm{NMR}, \mathrm{CDCl}_{3}, 90 \mathrm{MHz}$

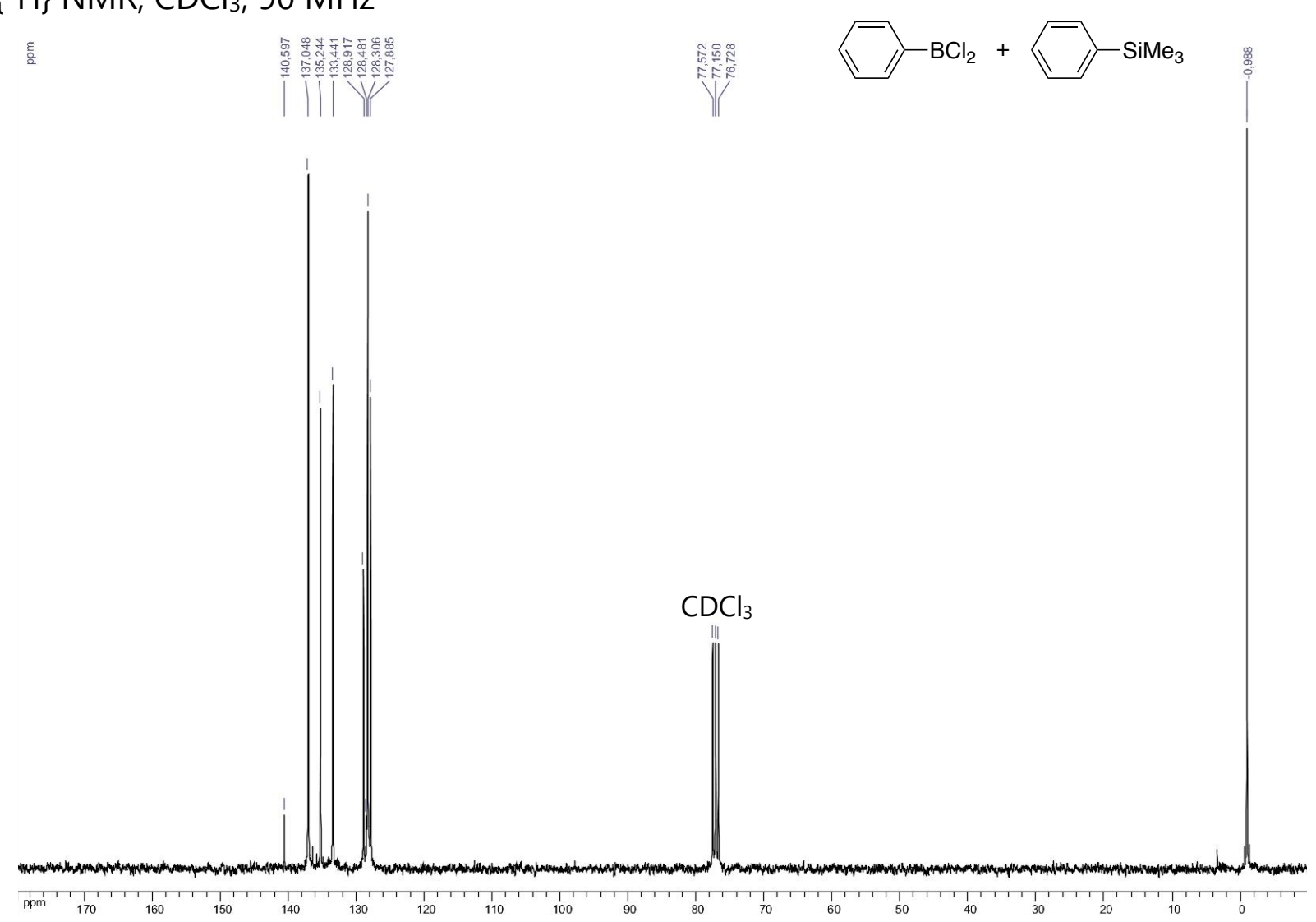



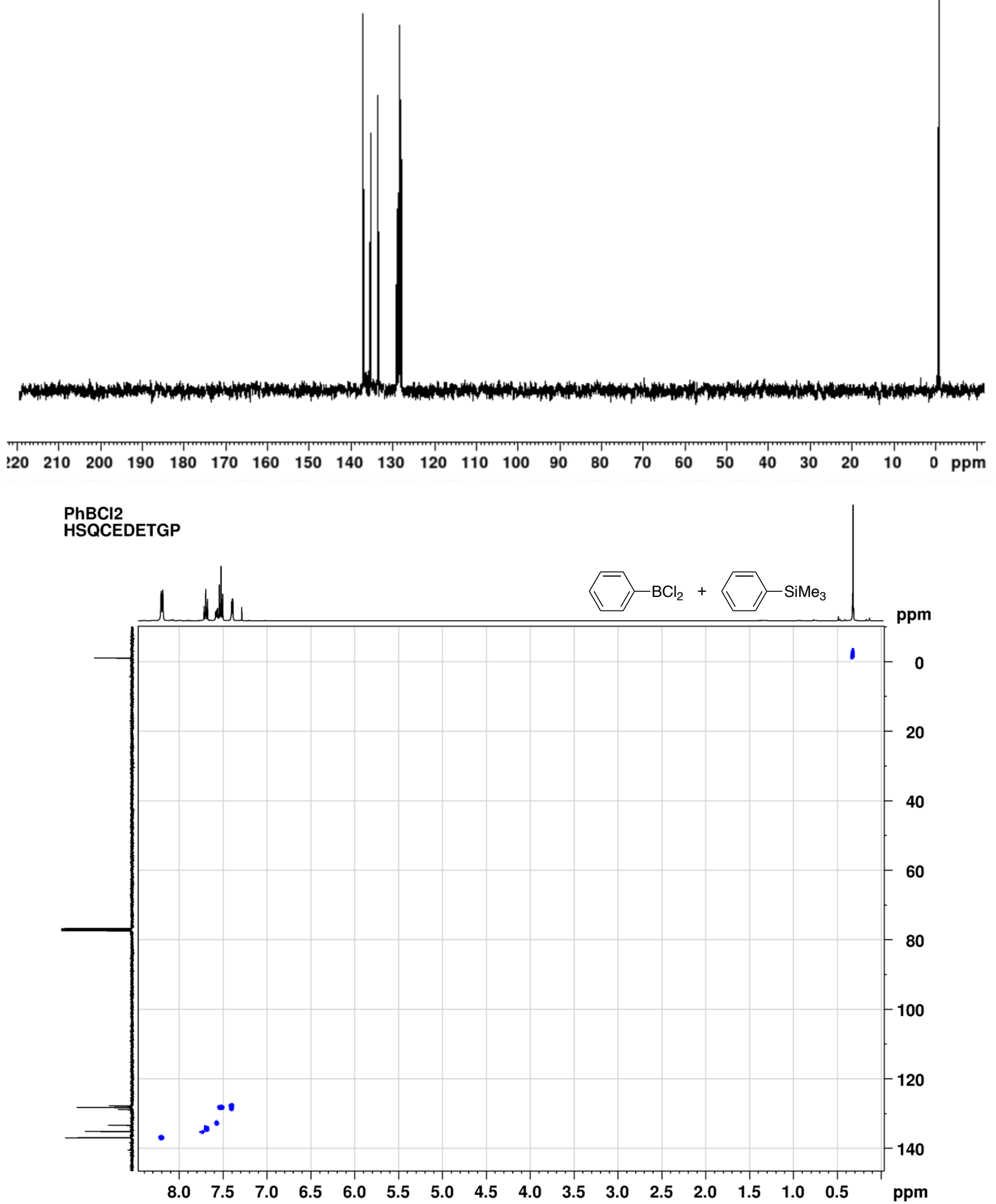

${ }^{11} \mathrm{~B} N M R, \mathrm{CDCl}_{3}, 96 \mathrm{MHz}$ 


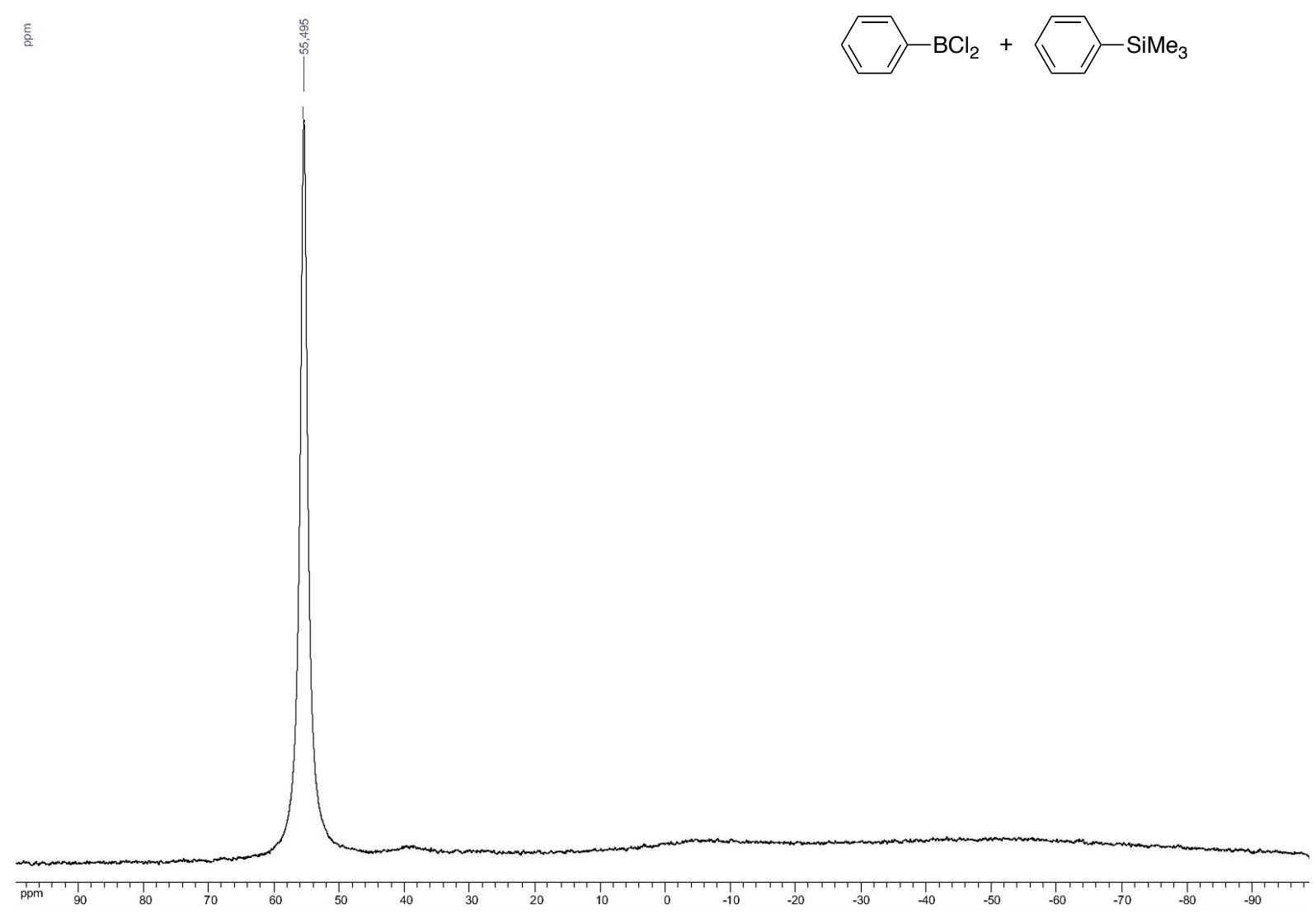


2. ${ }^{1} H$ and ${ }^{13} \mathrm{C}-\left\{{ }^{1} \mathrm{H}\right\}$ NMR data for compounds $3 a, 4 a, 5 a, 5 b, 6 a, 6 b, 8 a$ and $8 b$ 2.1. Allyl 2-azido-3,4-di-O-benzyl-2-deoxy- $\alpha$-D-glucopyranoside (3a)

${ }^{1} \mathrm{H}$ NMR, $\mathrm{CDCl}_{3}, 360 \mathrm{MHz}$

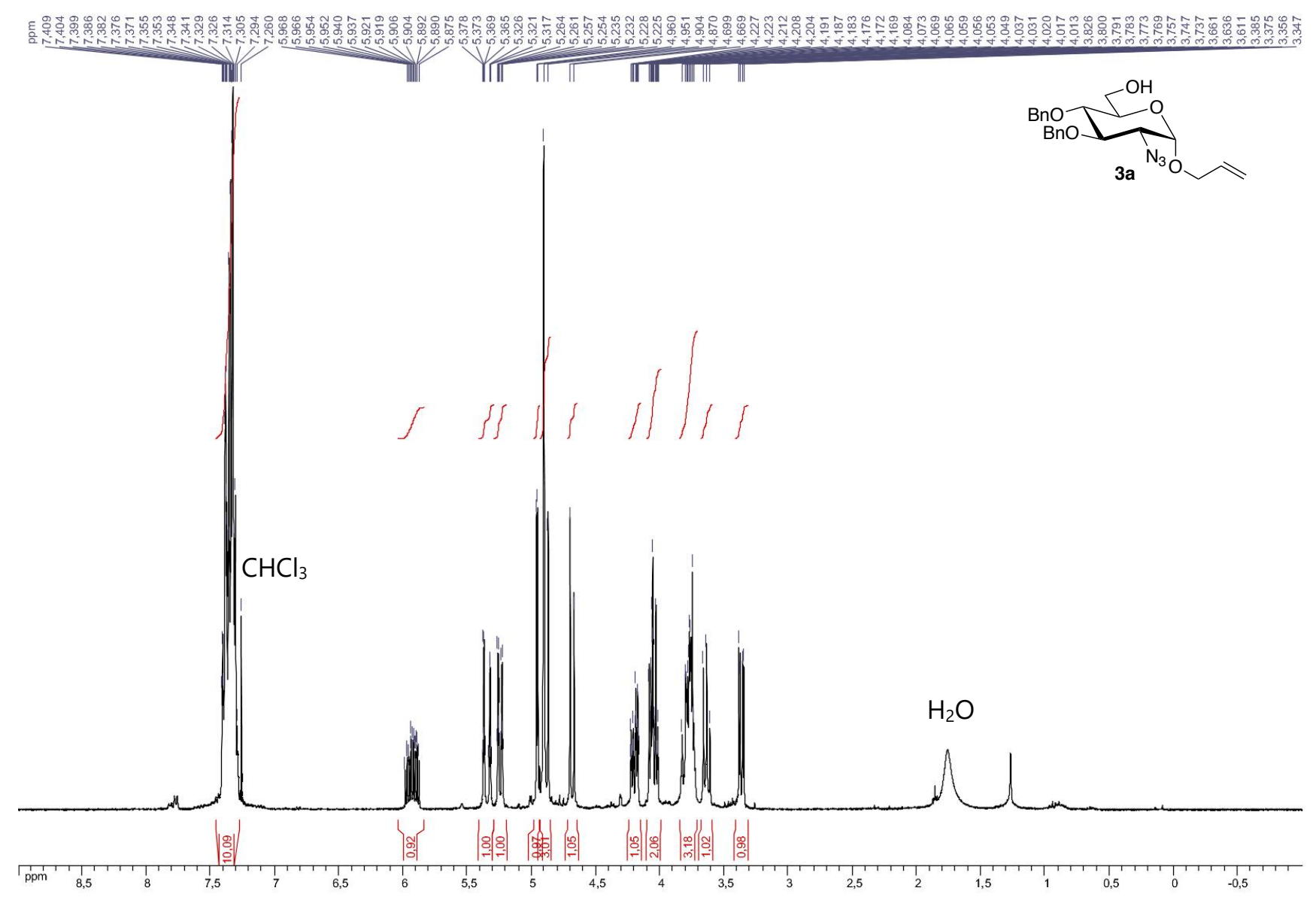

${ }^{13} \mathrm{C}-\left\{{ }^{1} \mathrm{H}\right\} \mathrm{NMR}, \mathrm{CDCl}_{3}, 90 \mathrm{MHz}$

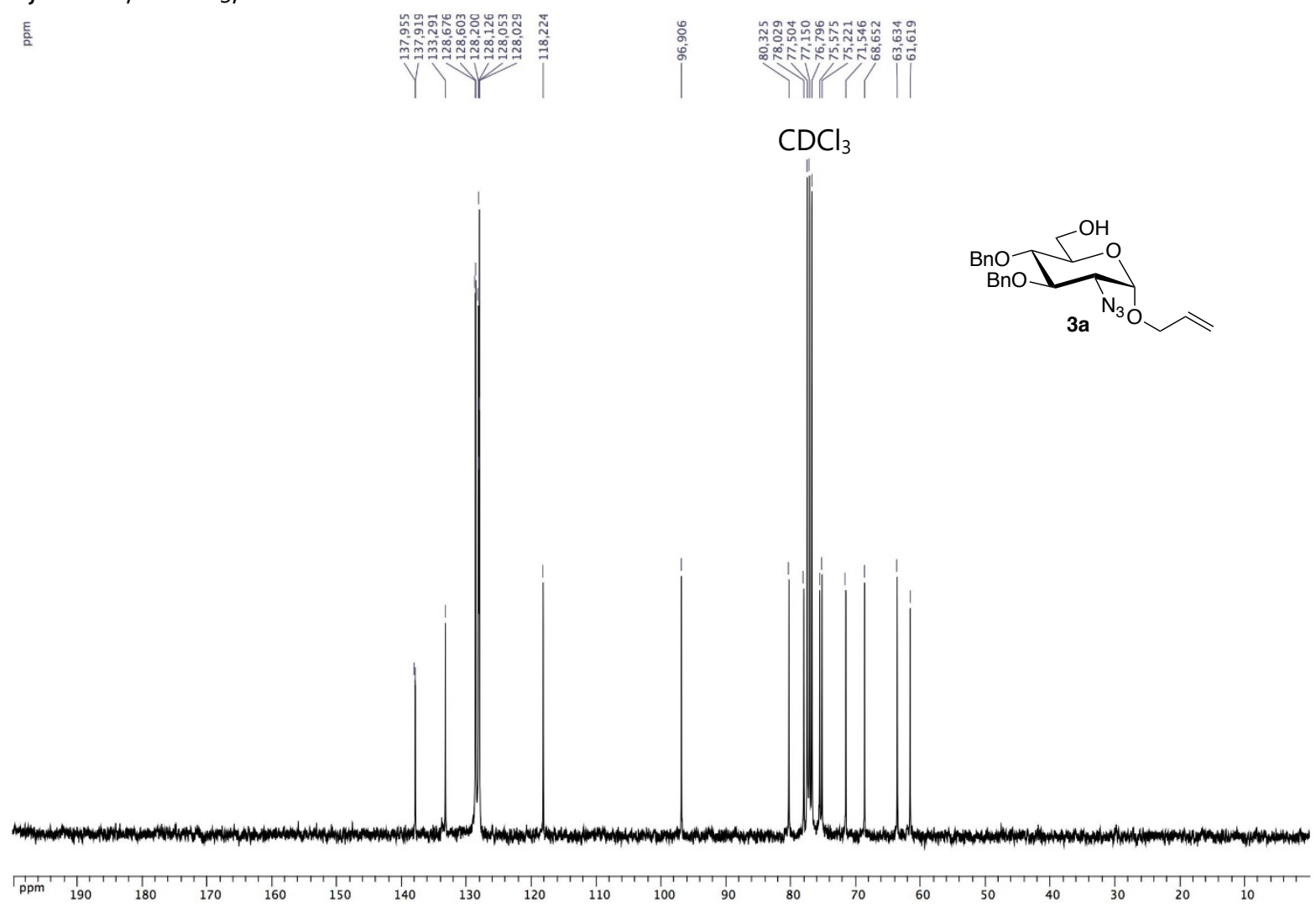


Compound 3a

CDCl3

DEPT135

$\left.\right|^{\mid}$
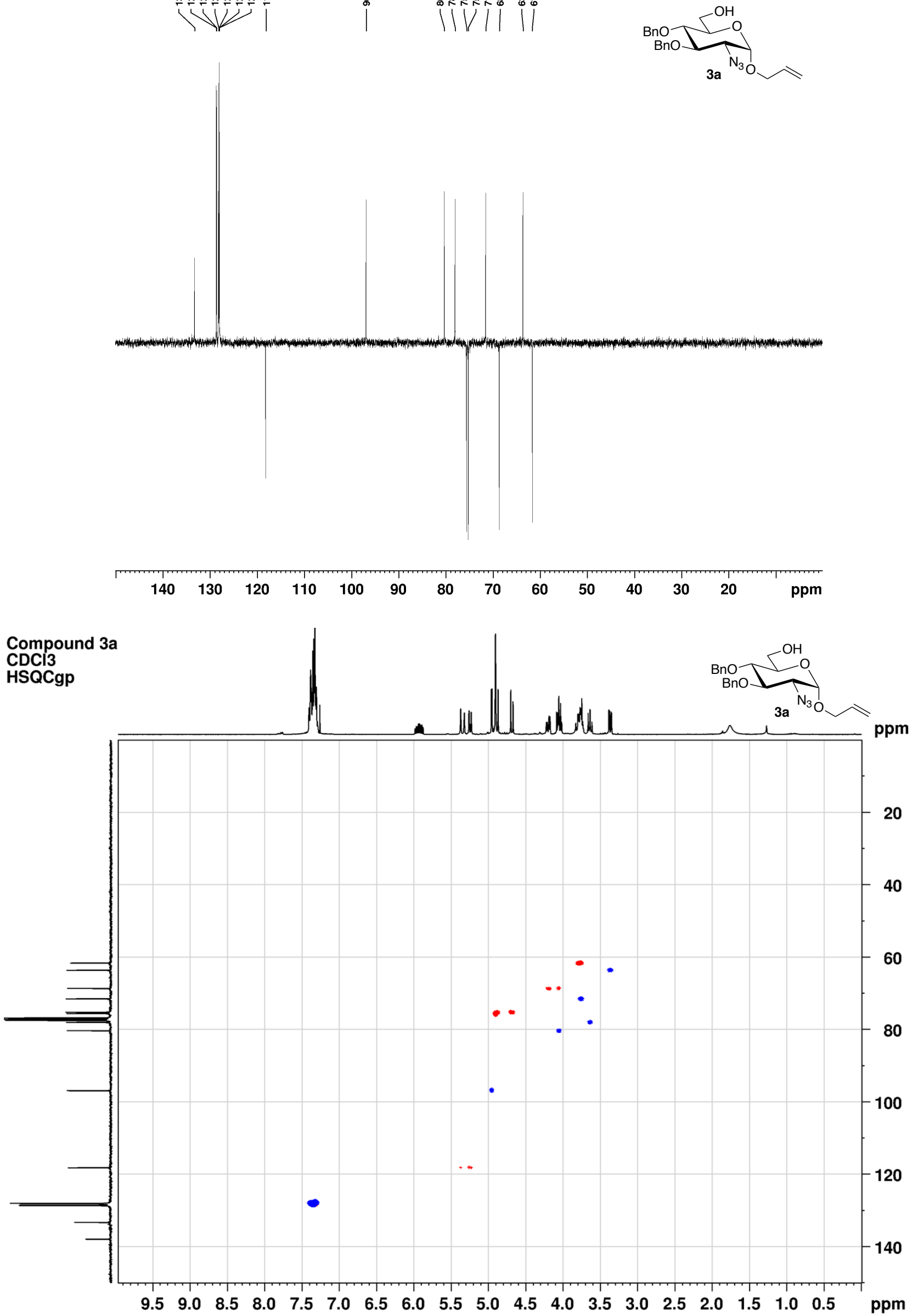
2.2. Allyl (2-azido-3,4-di-O-benzyl-2-deoxy- $\alpha$-D-glucopyranosyl)-(1 $\rightarrow 4)-($ methyl 2-O-acetyl-3-O-benzyl-2-deoxy$\alpha$-L-idopyranuronyl)-(1 $\rightarrow 4)-6-0$-acetyl-2-azido-3-O-benzyl-2-deoxy- $\alpha$-D-glucopyranoside (4a)

${ }^{1} \mathrm{H} \mathrm{NMR}, \mathrm{CDCl}_{3}, 300 \mathrm{MHz}$

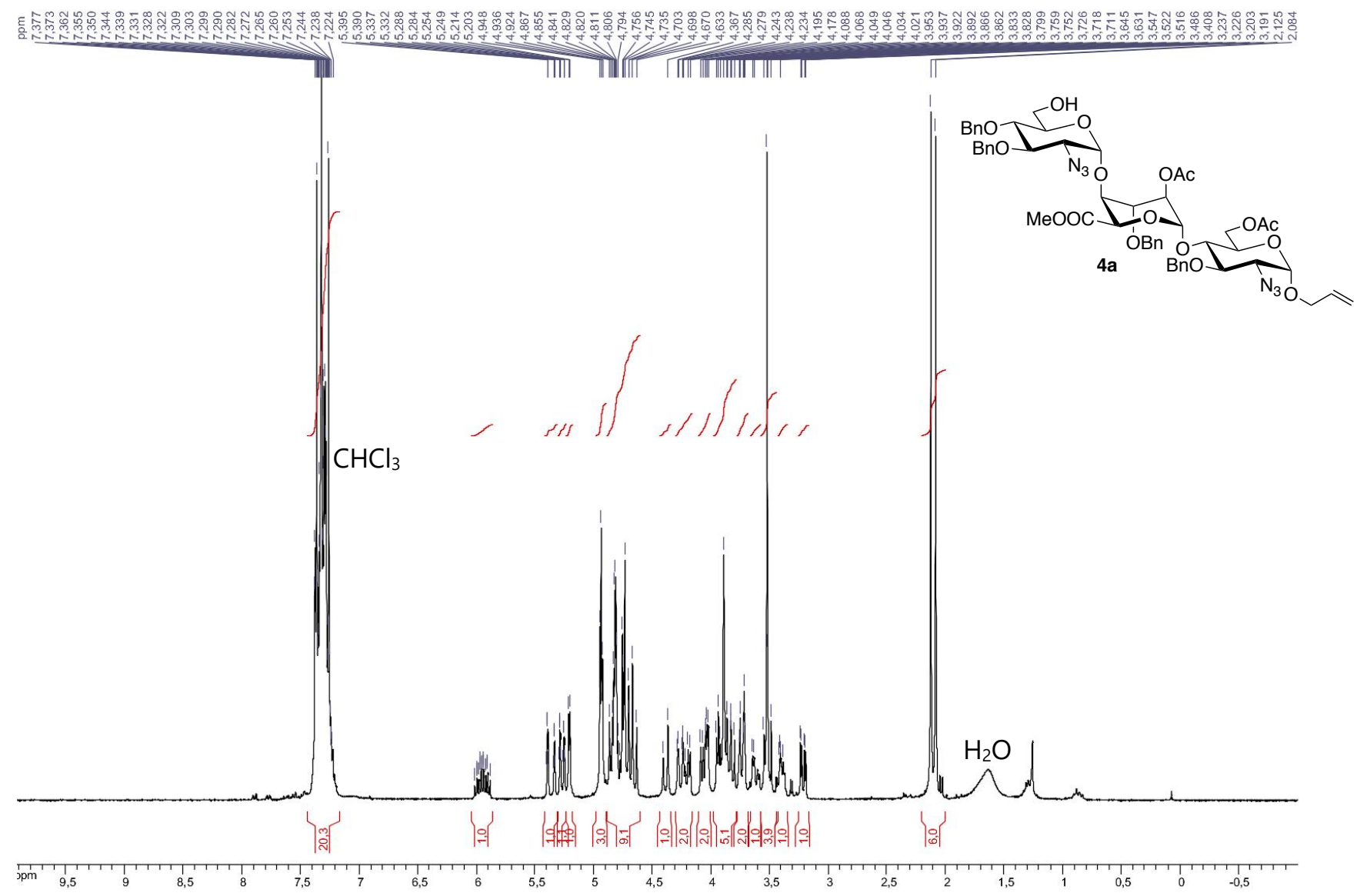

${ }^{13} \mathrm{C}-\left\{{ }^{1} \mathrm{H}\right\} \mathrm{NMR}, \mathrm{CDCl}_{3}, 75 \mathrm{MHz}$

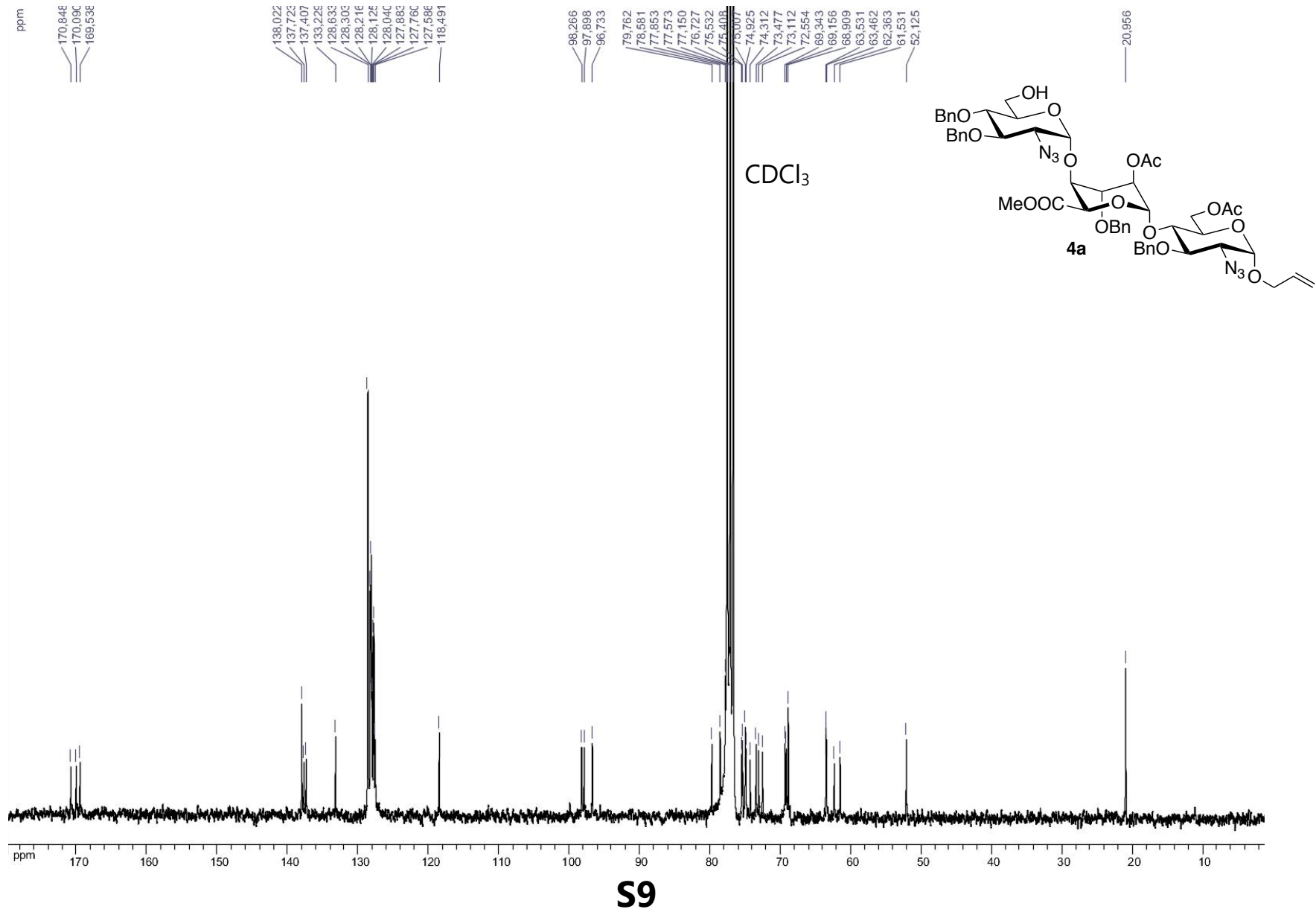




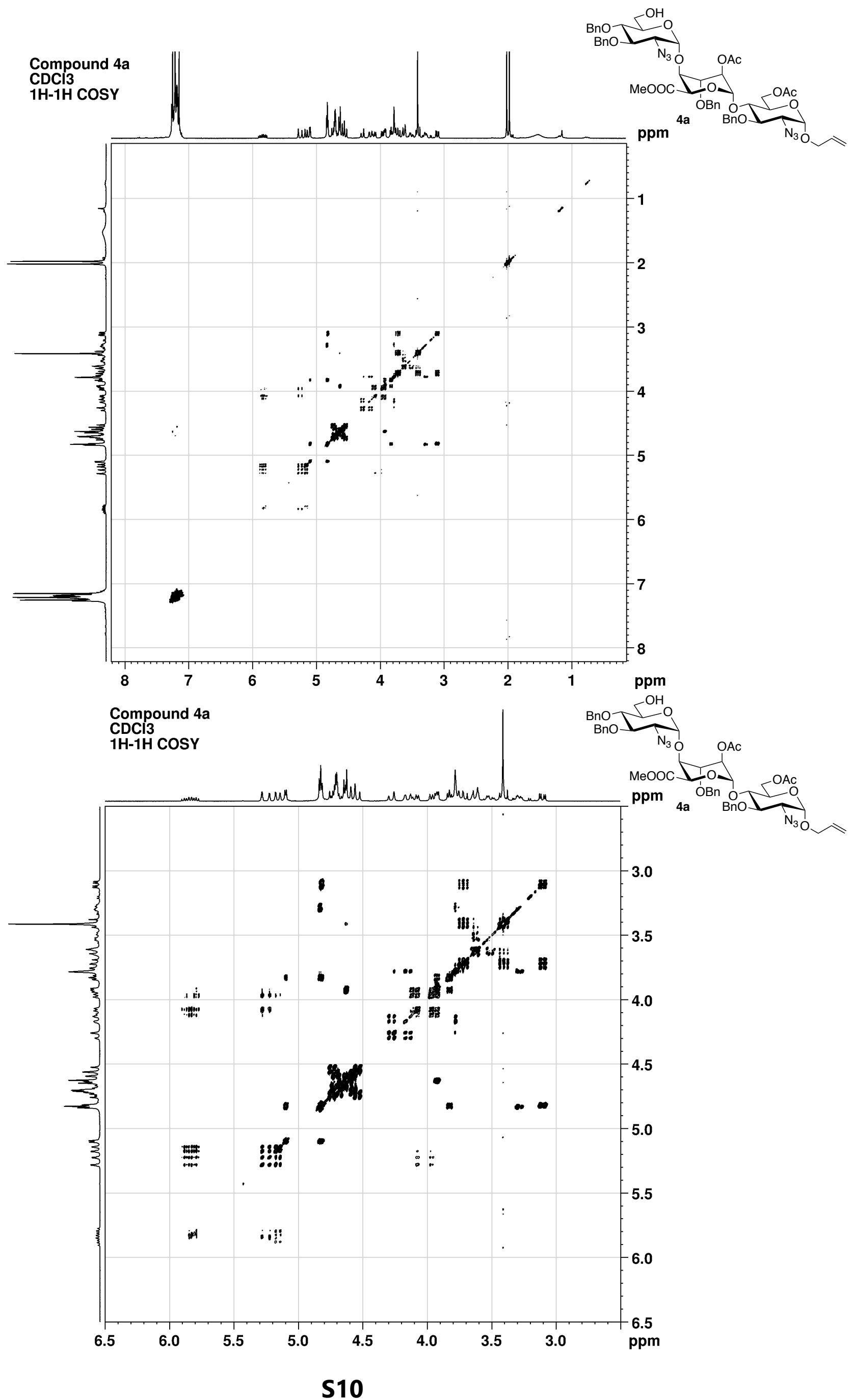



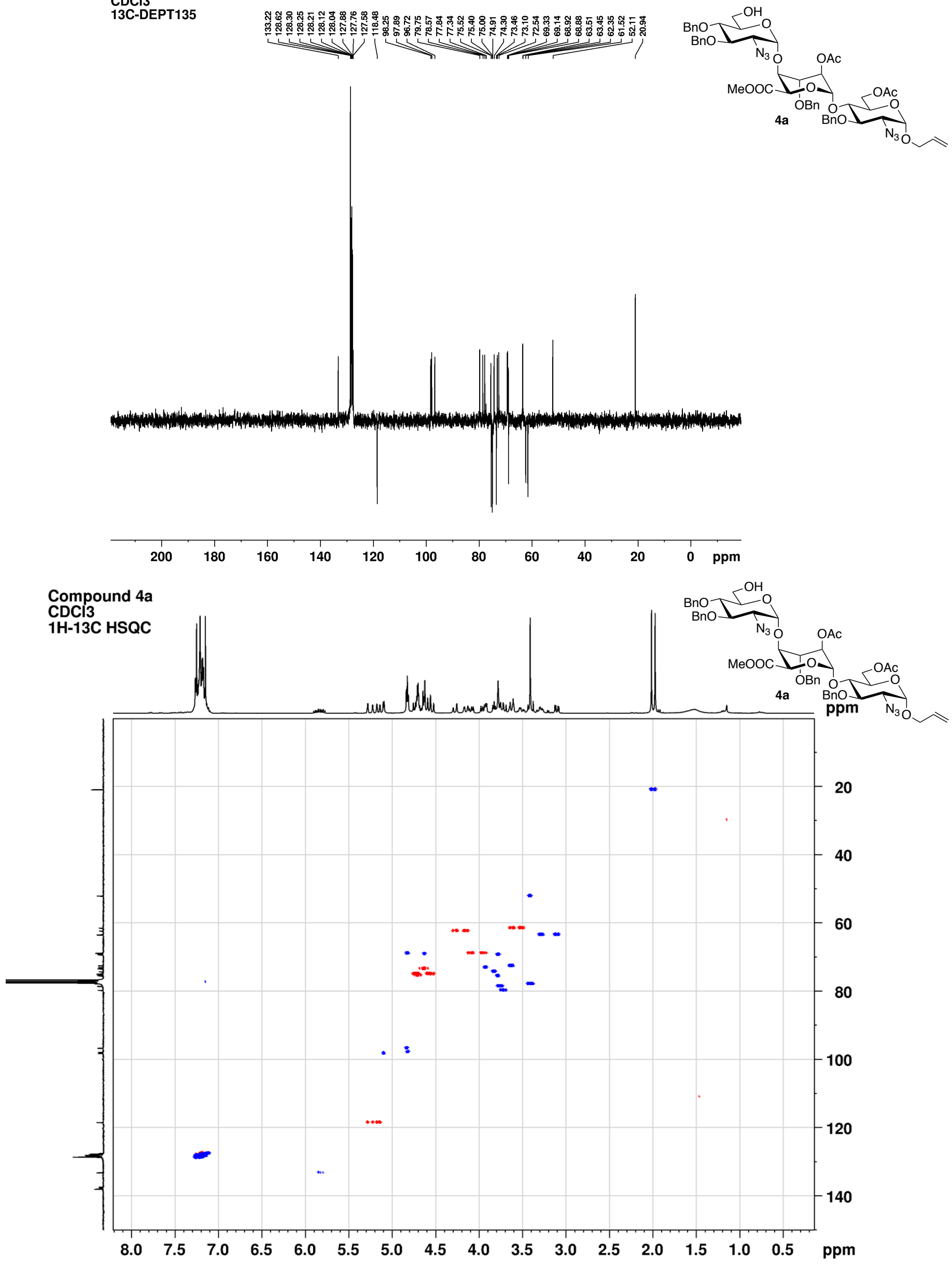
2.3. 3-hydroxypropyl 2-azido-3,4-di-O-benzyl-2-deoxy- $\alpha$-D-glucopyranoside (5a) and 2-hydroxypropyl 2-azido3,4-di-O-benzyl-2-deoxy- $\alpha$-D-glucopyranoside (5b).

${ }^{1} \mathrm{H} N M R, \mathrm{CDCl}_{3}, 360 \mathrm{MHz}$

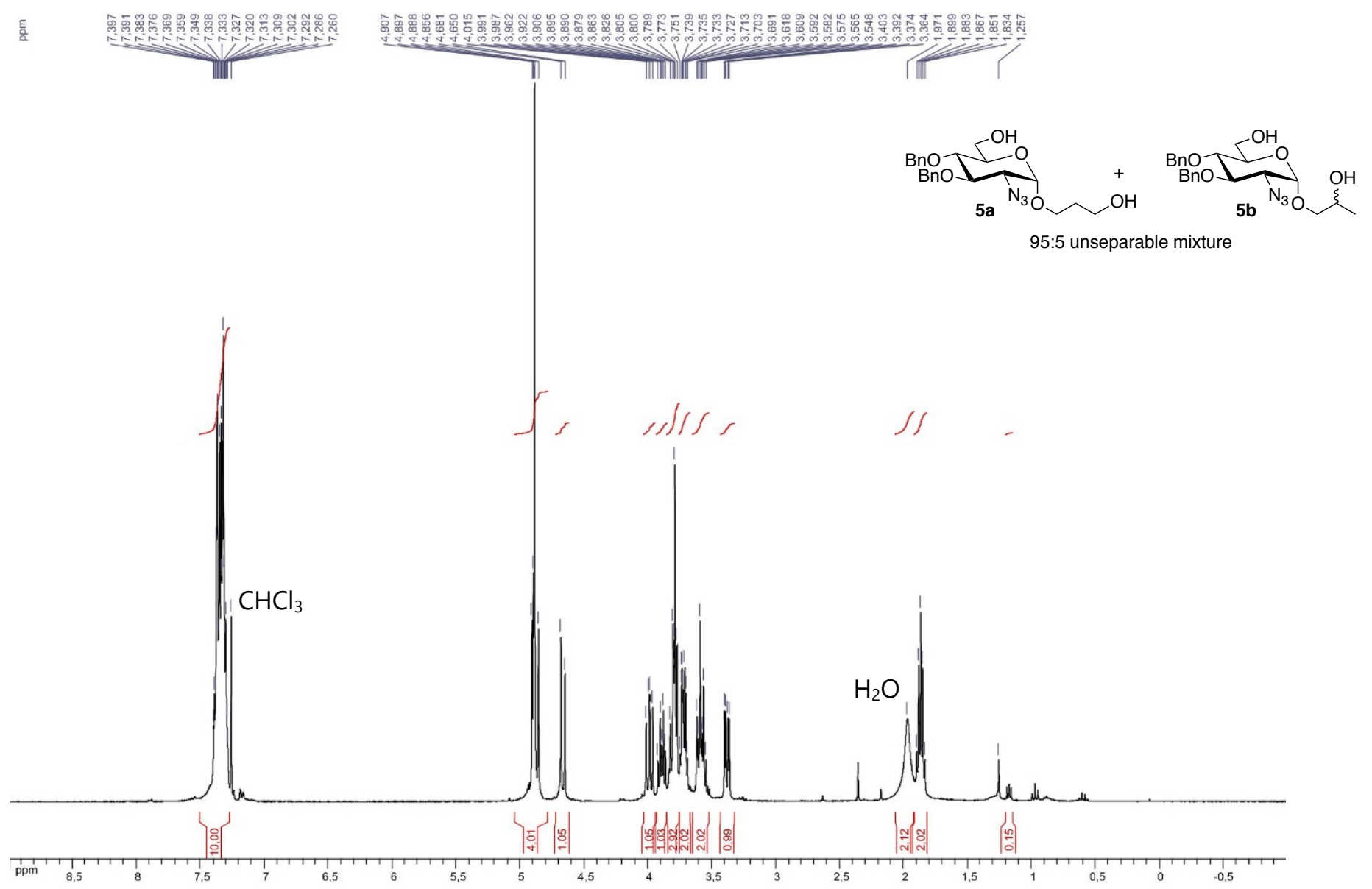

${ }^{13} \mathrm{C}-\left\{{ }^{1} \mathrm{H}\right\} \mathrm{NMR}, \mathrm{CDCl}_{3}, 90 \mathrm{MHz}$

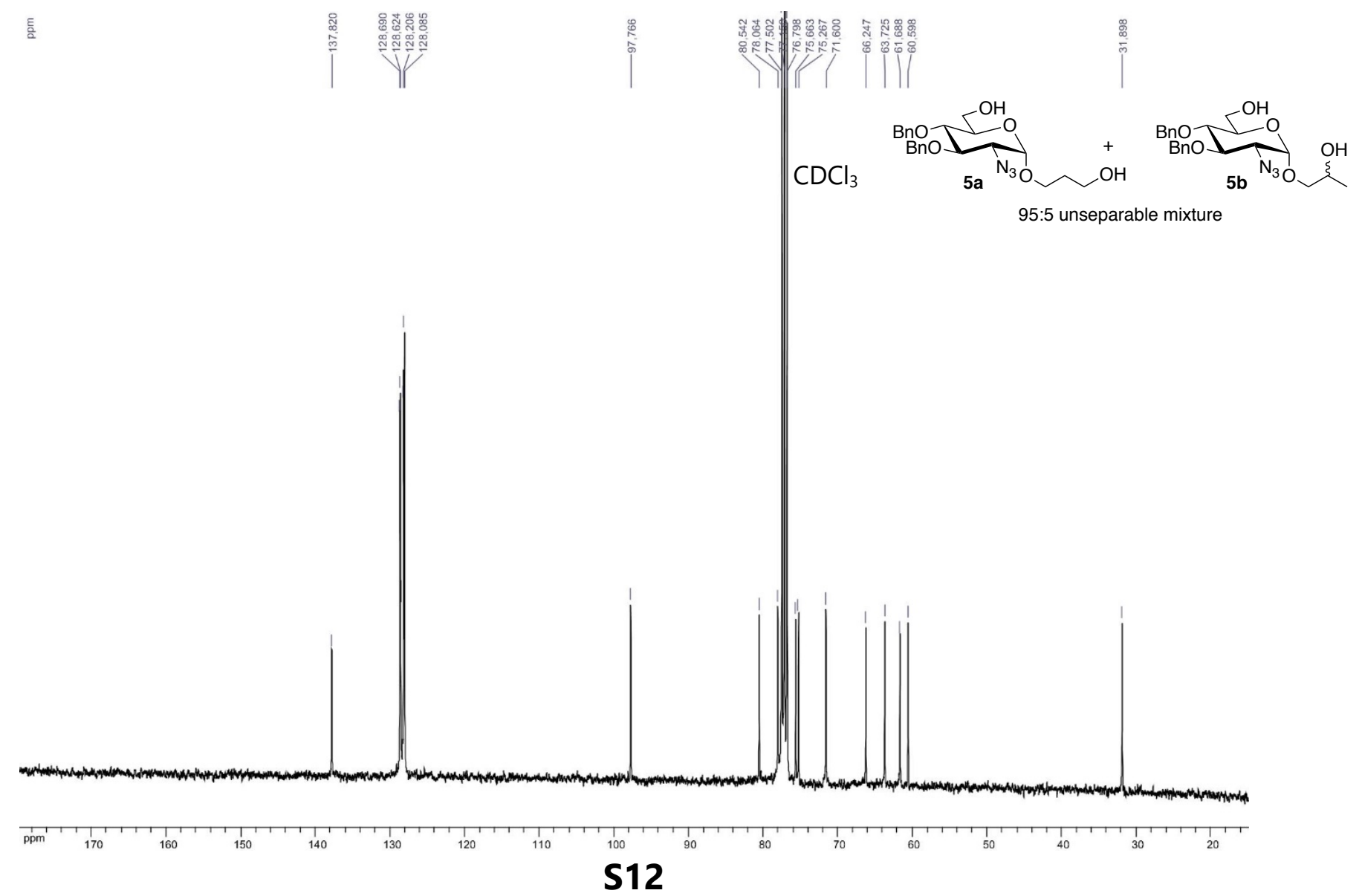



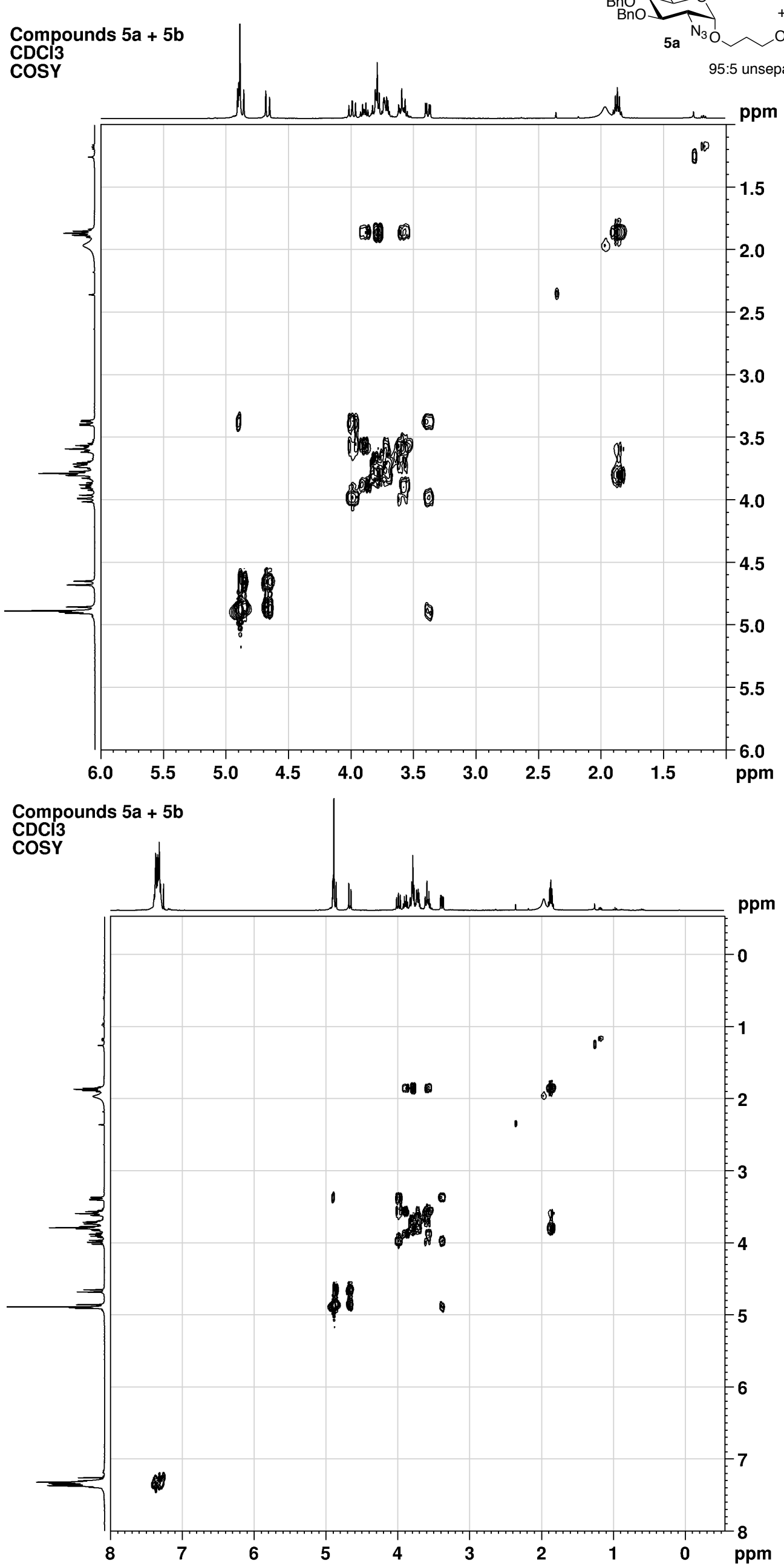


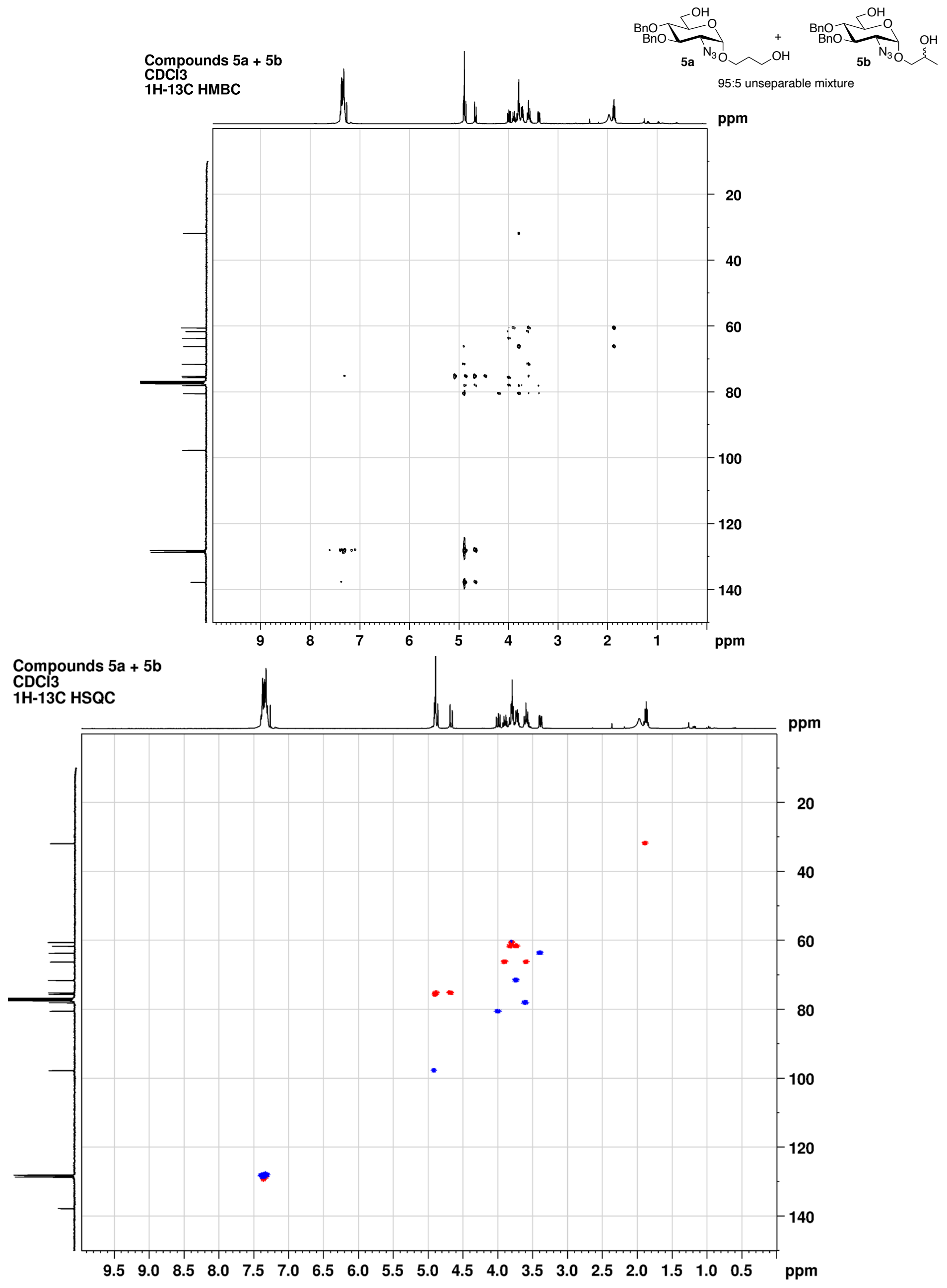


2.4. 3-hydroxypropyl (2-azido-3,4-di-O-benzyl-2-deoxy- $\alpha$-D-glucopyranosyl)-(1->4)-(methyl 2-O-acetyl-3-0benzyl-2-deoxy- $\alpha$-L-idopyranuronyl)-(1->4)-6-0-acetyl-2-azido-3-O-benzyl-2-deoxy- $\alpha$-D-glucopyranoside (6a) ${ }^{1} \mathrm{H} N M R, \mathrm{CDCl}_{3}, 400 \mathrm{MHz}$

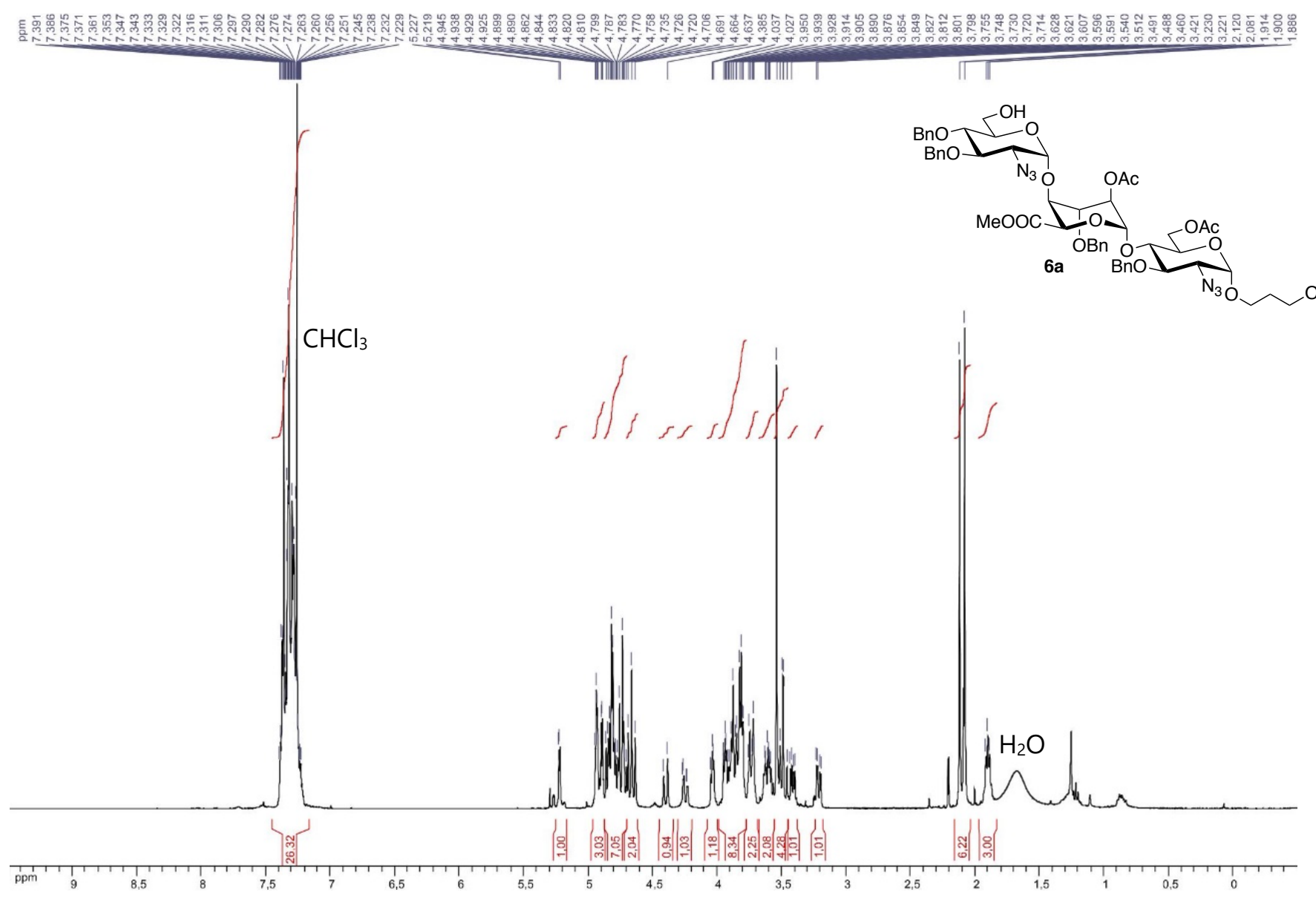

${ }^{13} \mathrm{C}-\left\{{ }^{1} \mathrm{H}\right\} \mathrm{NMR}, \mathrm{CDCl}_{3}, 90 \mathrm{MHz}$

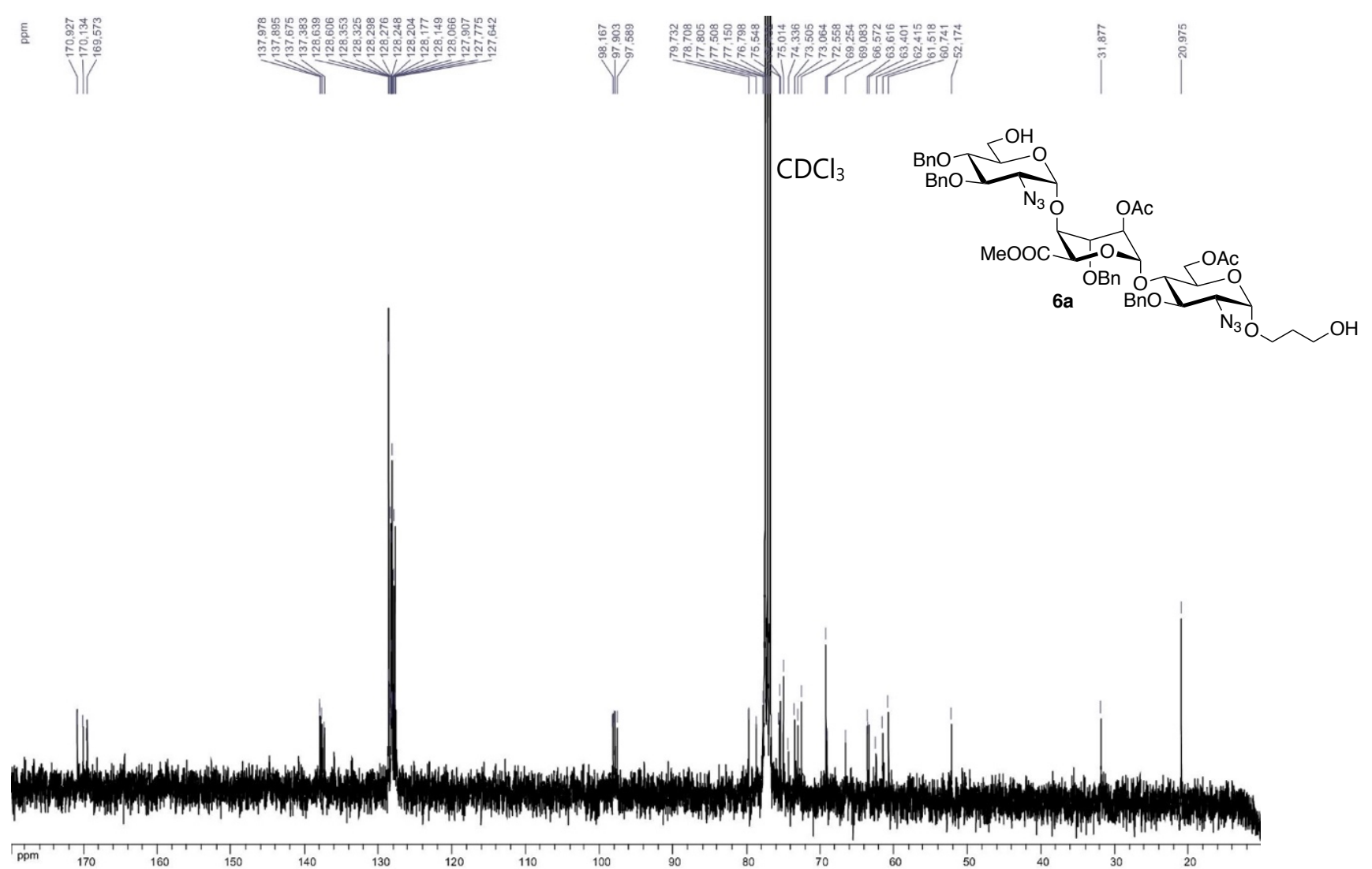




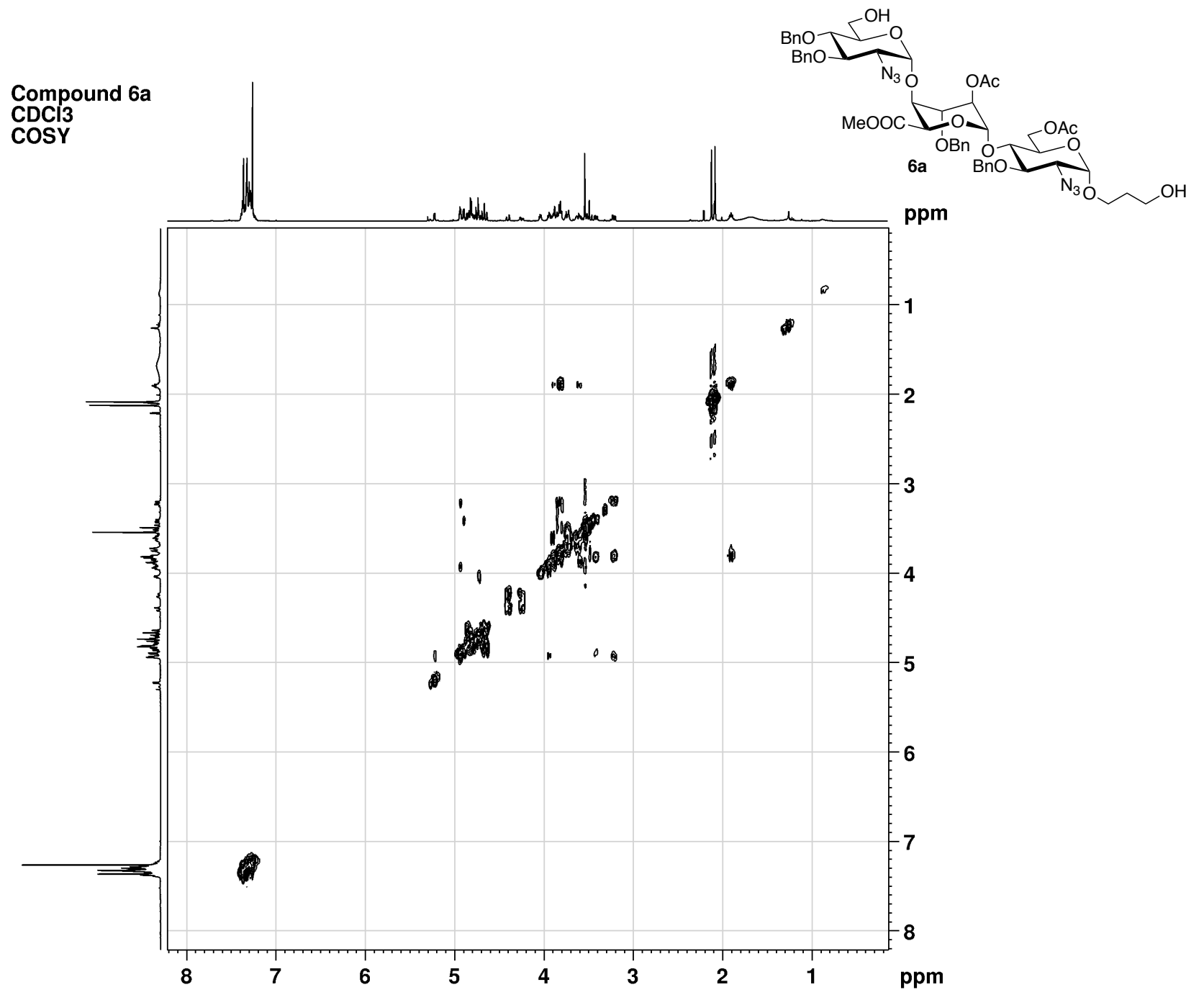

Compound 6a

CDCl3

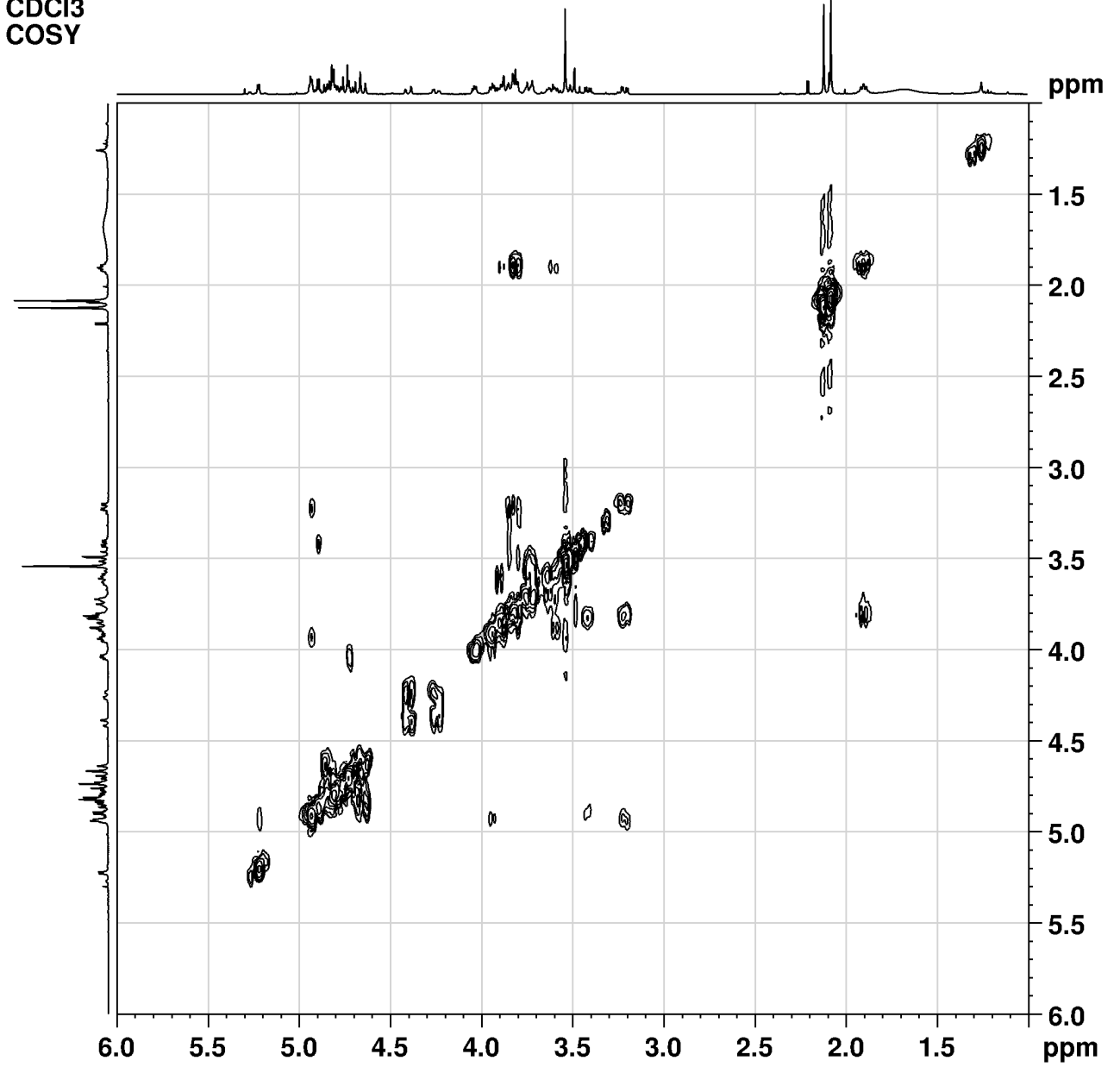



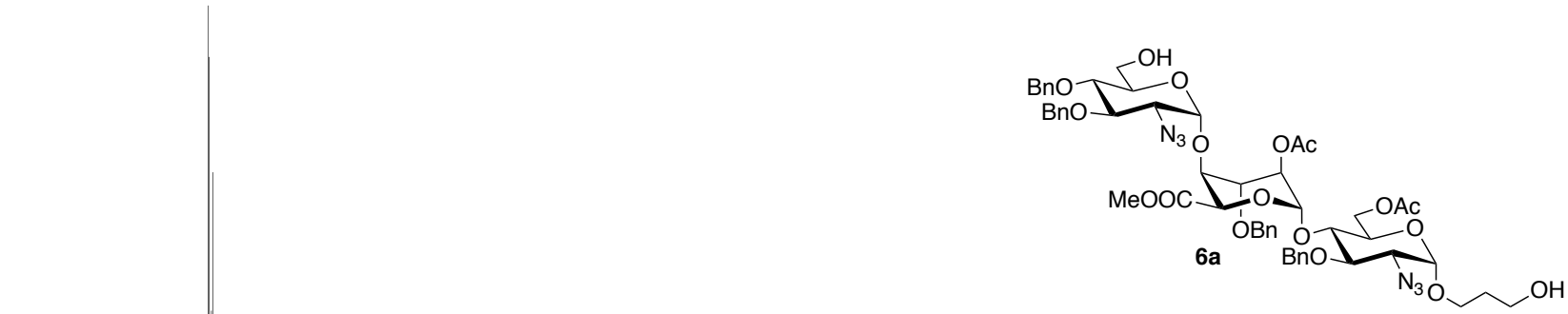

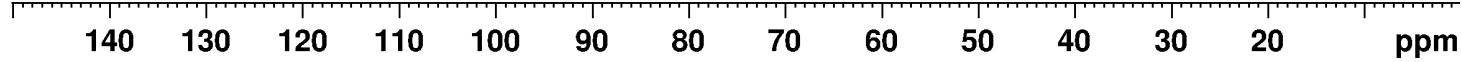

Compound $6 a$ CDC13

$1 \mathrm{H}-13 \mathrm{C} \mathrm{HSQC}$

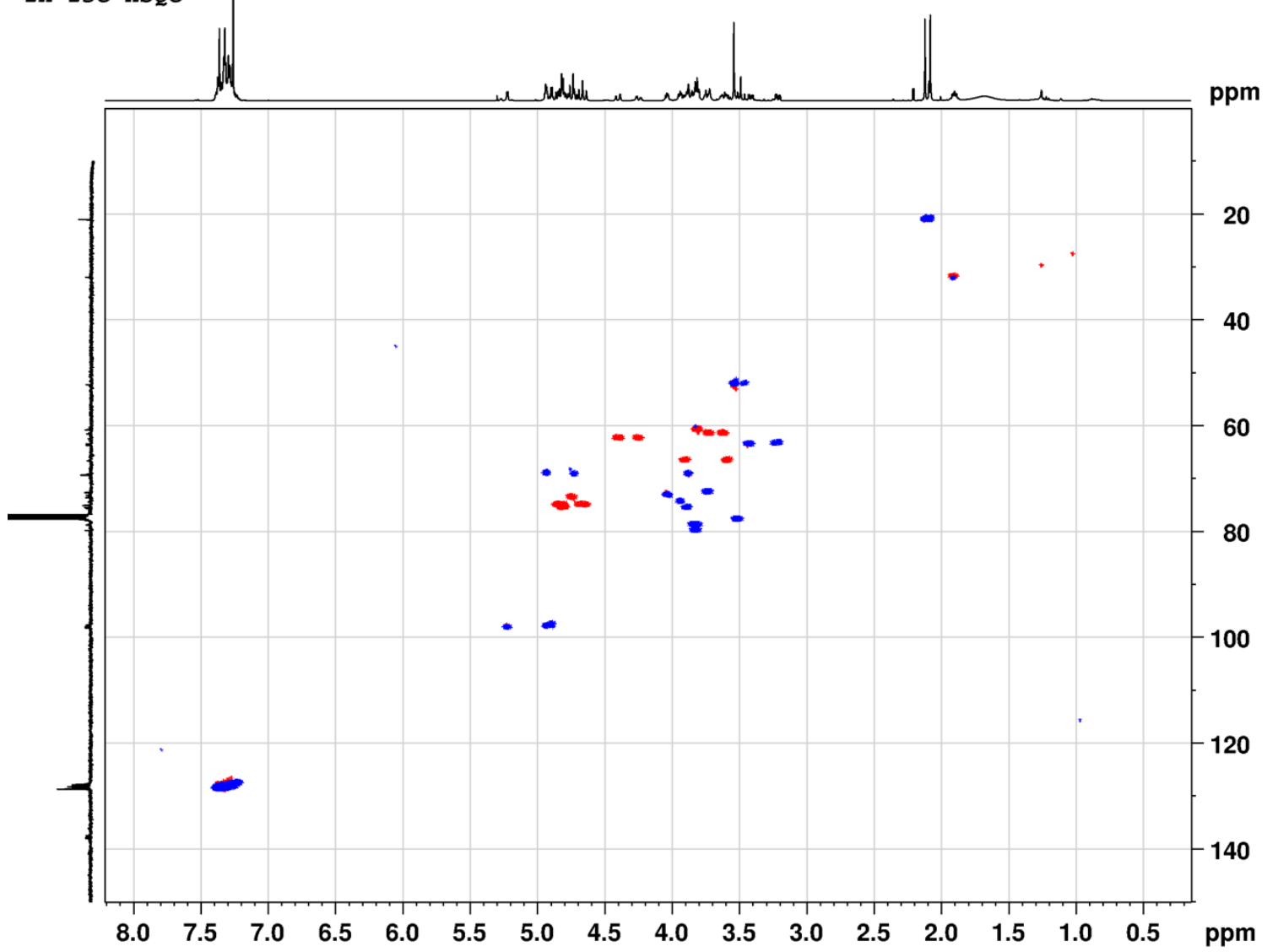


2.5. 2-hydroxypropyl (2-azido-3,4-di-O-benzyl-2-deoxy- $\alpha$-D-glucopyranosyl)-(1->4)-(methyl 2-O-acetyl-3-Obenzyl-2-deoxy- $\alpha$-L-idopyranuronyl)-(1->4)-6-0-acetyl-2-azido-3-O-benzyl-2-deoxy- $\alpha$-D-glucopyranoside (6b) ${ }^{1} \mathrm{H} N M R, \mathrm{CDCl}_{3}, 400 \mathrm{MHz}$

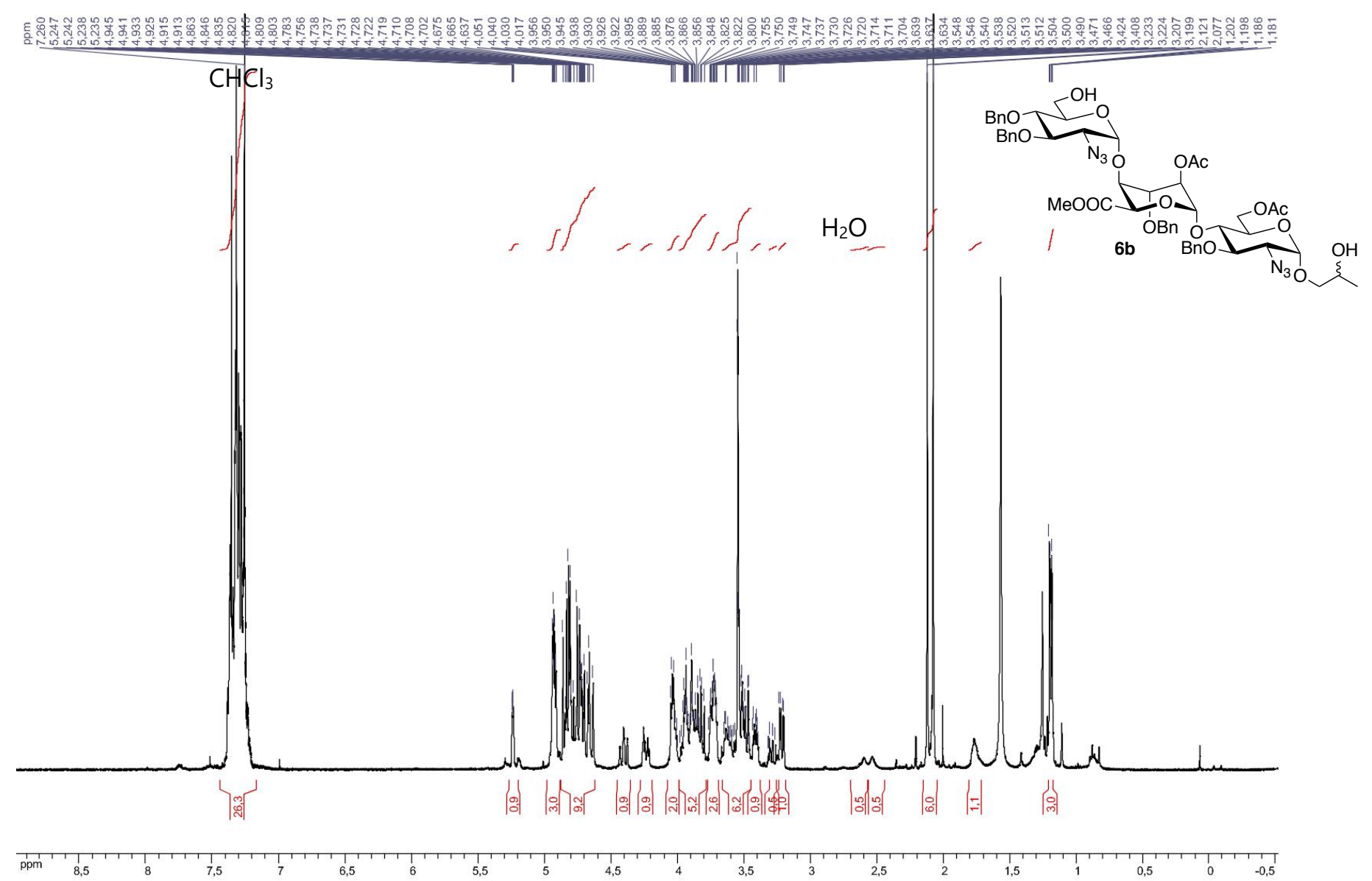

${ }^{13} \mathrm{C}-\left\{{ }^{1} \mathrm{H}\right\}$ and DEPT-135 NMR, $\mathrm{CDCl}_{3}, 90 \mathrm{MHz}$

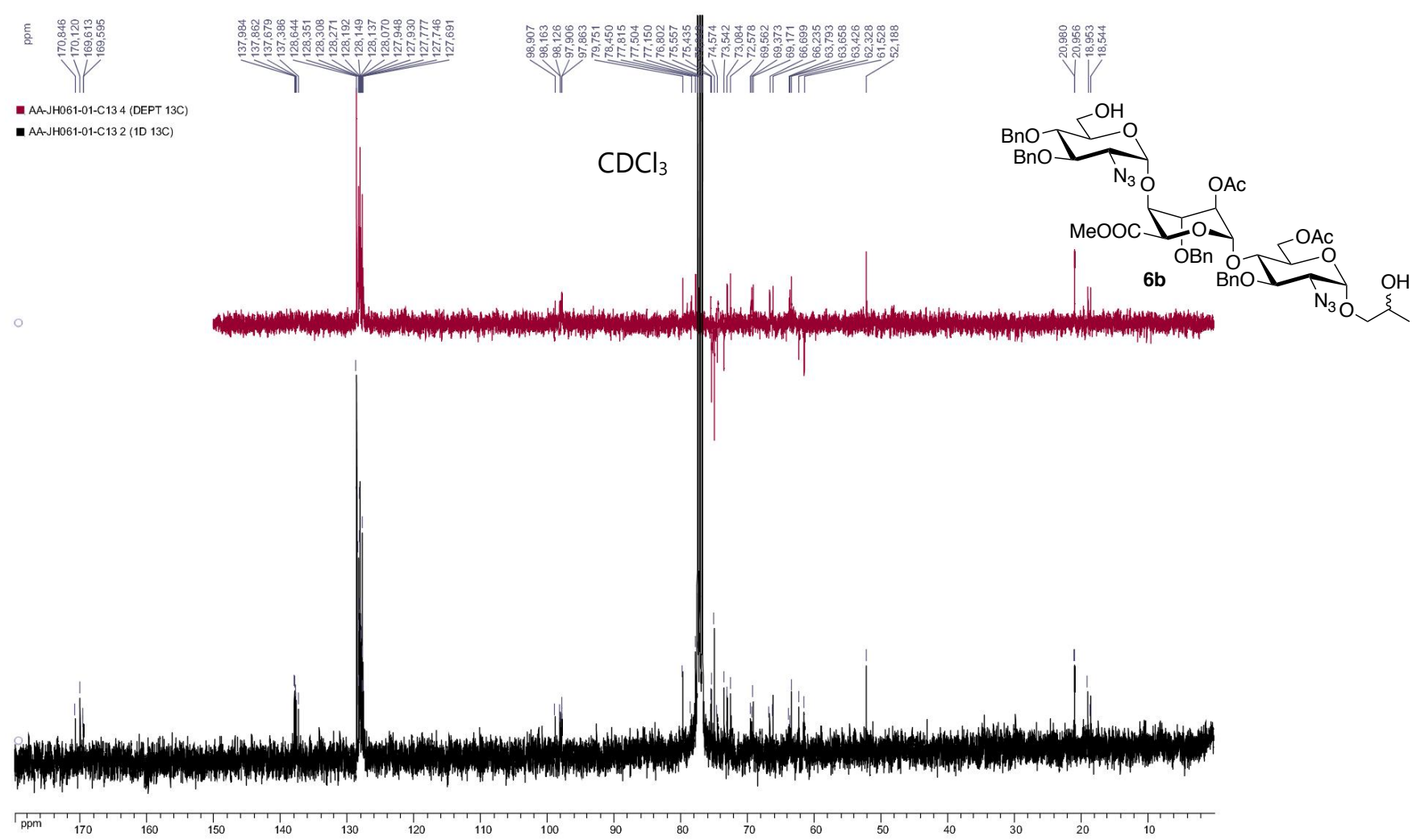




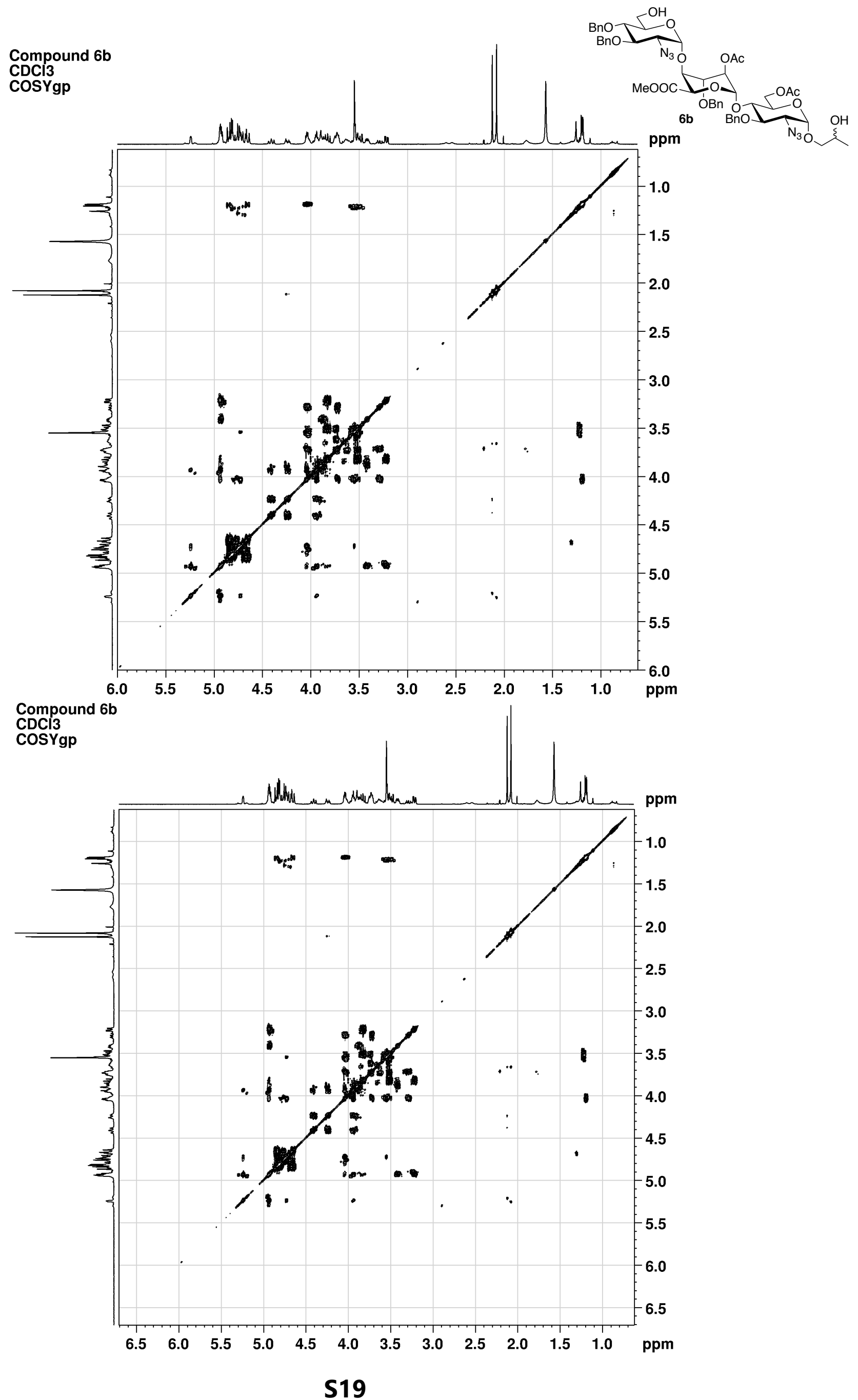




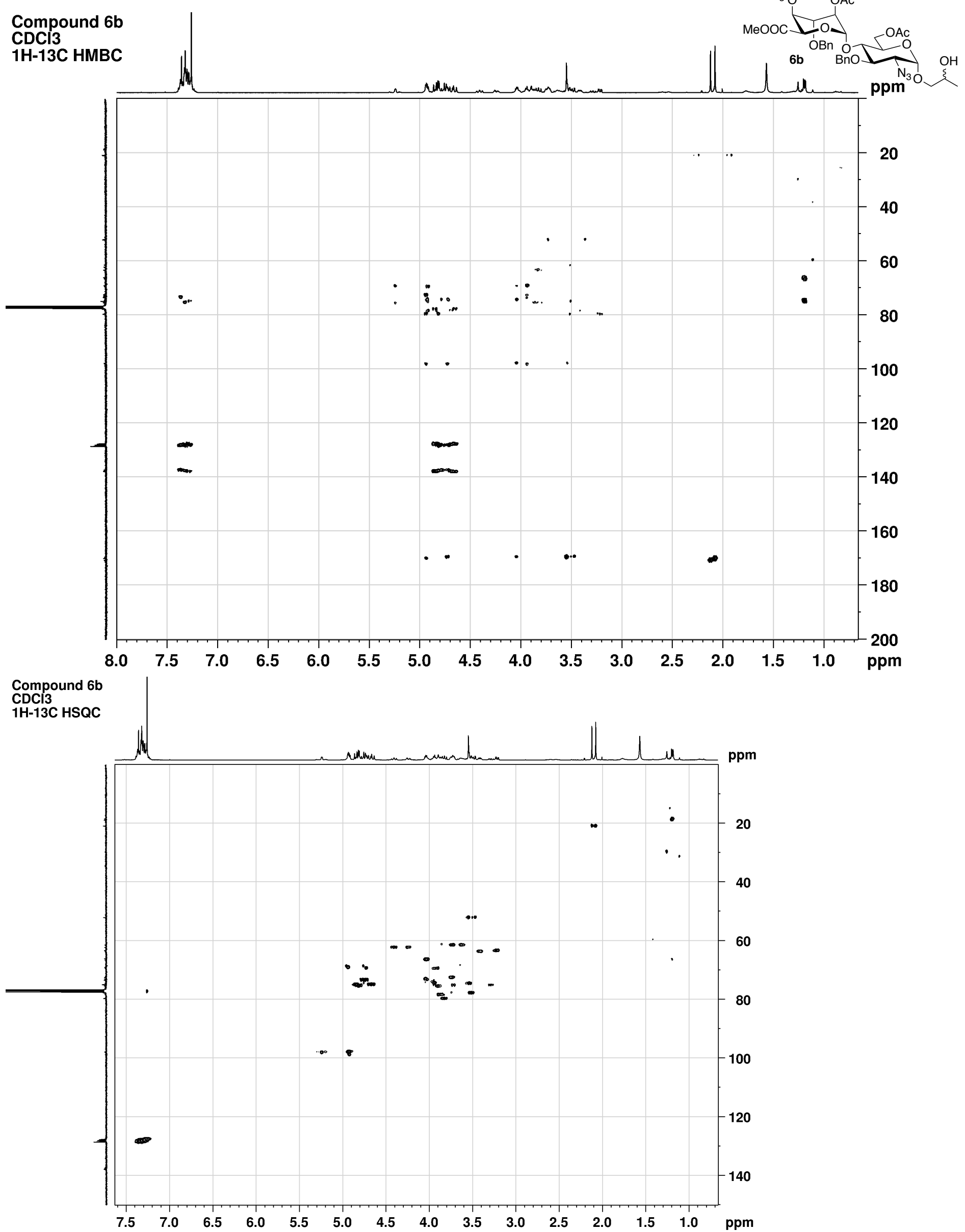


2.6. 3-hydroxypropyl 2-azido-3,4,6-tri-O-benzyl-2-deoxy- $\alpha$-D-glucopyranoside (8a)

${ }^{1} \mathrm{H} \mathrm{NMR}, \mathrm{CDCl}_{3}, 300 \mathrm{MHz}$

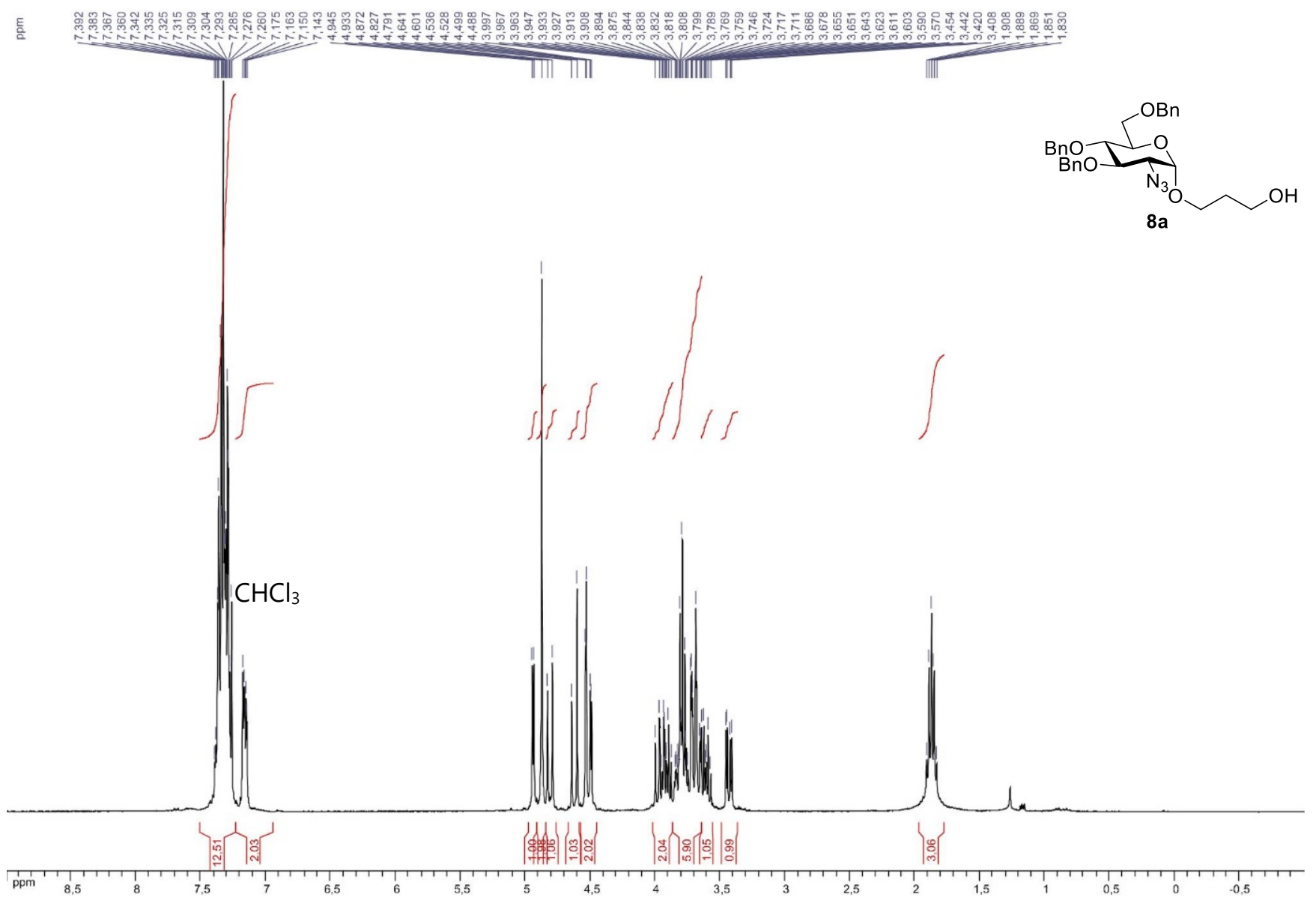

${ }^{13} \mathrm{C}-\left\{{ }^{1} \mathrm{H}\right\} \mathrm{NMR}, \mathrm{CDCl}_{3}, 75 \mathrm{MHz}$
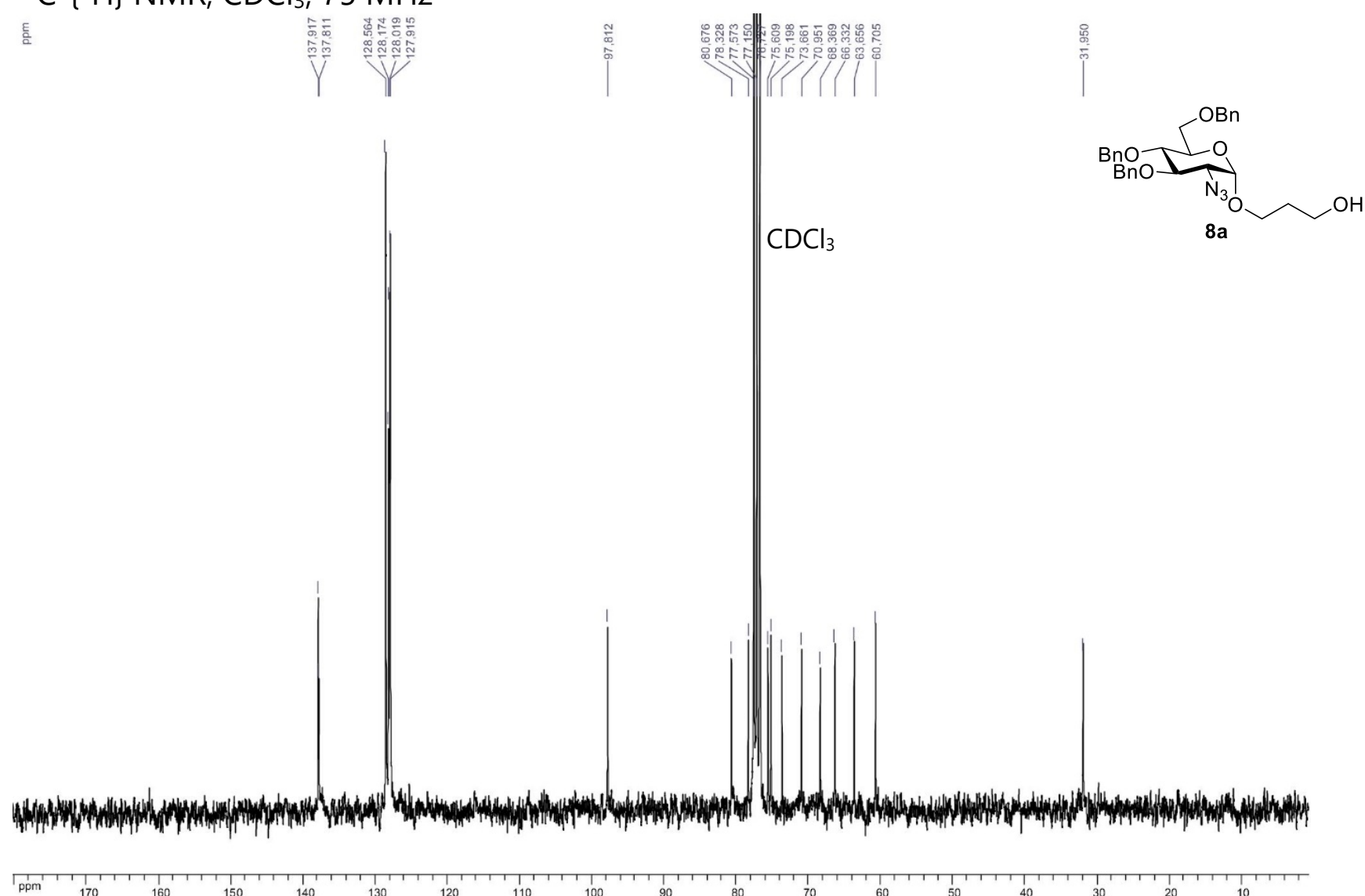

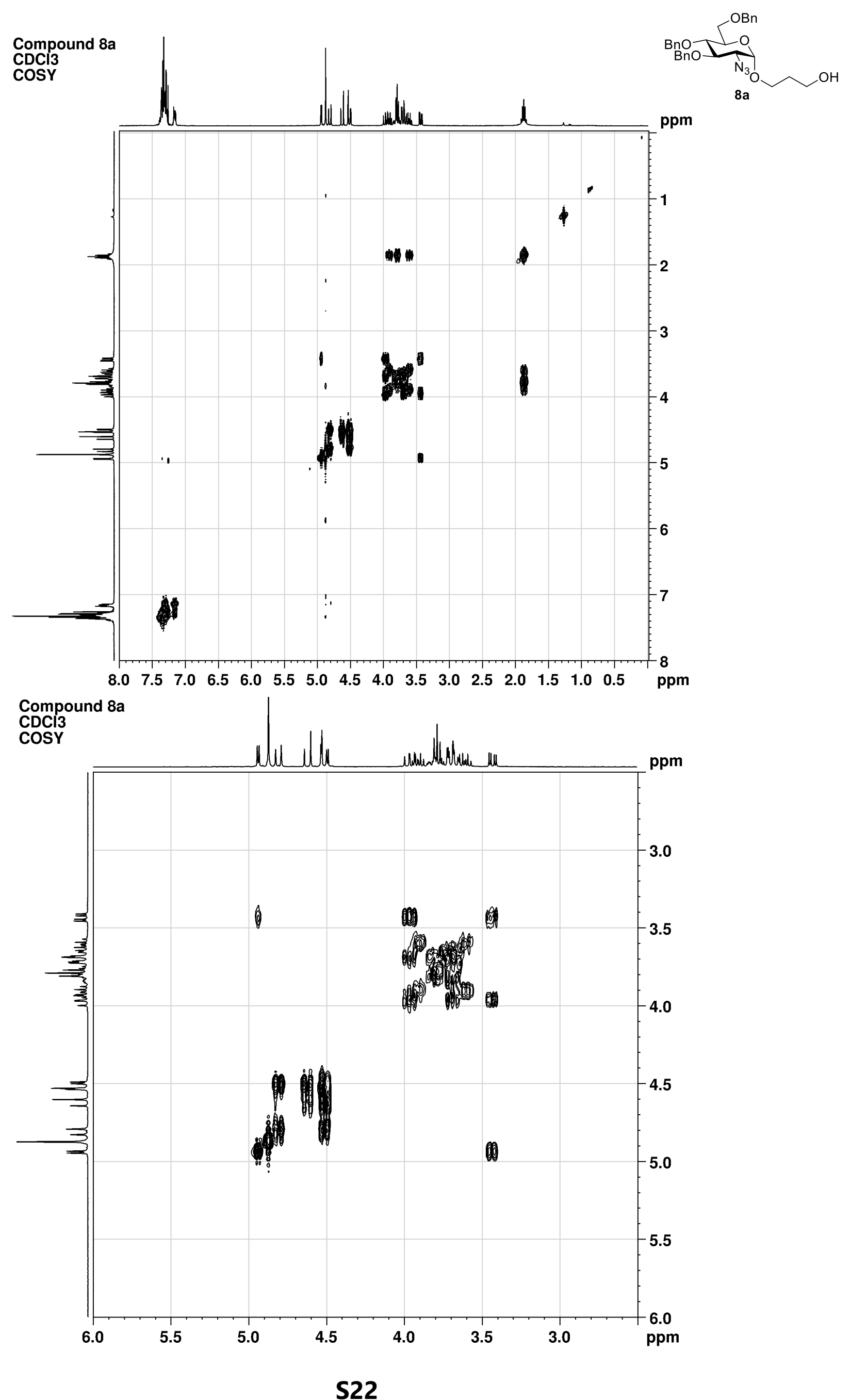

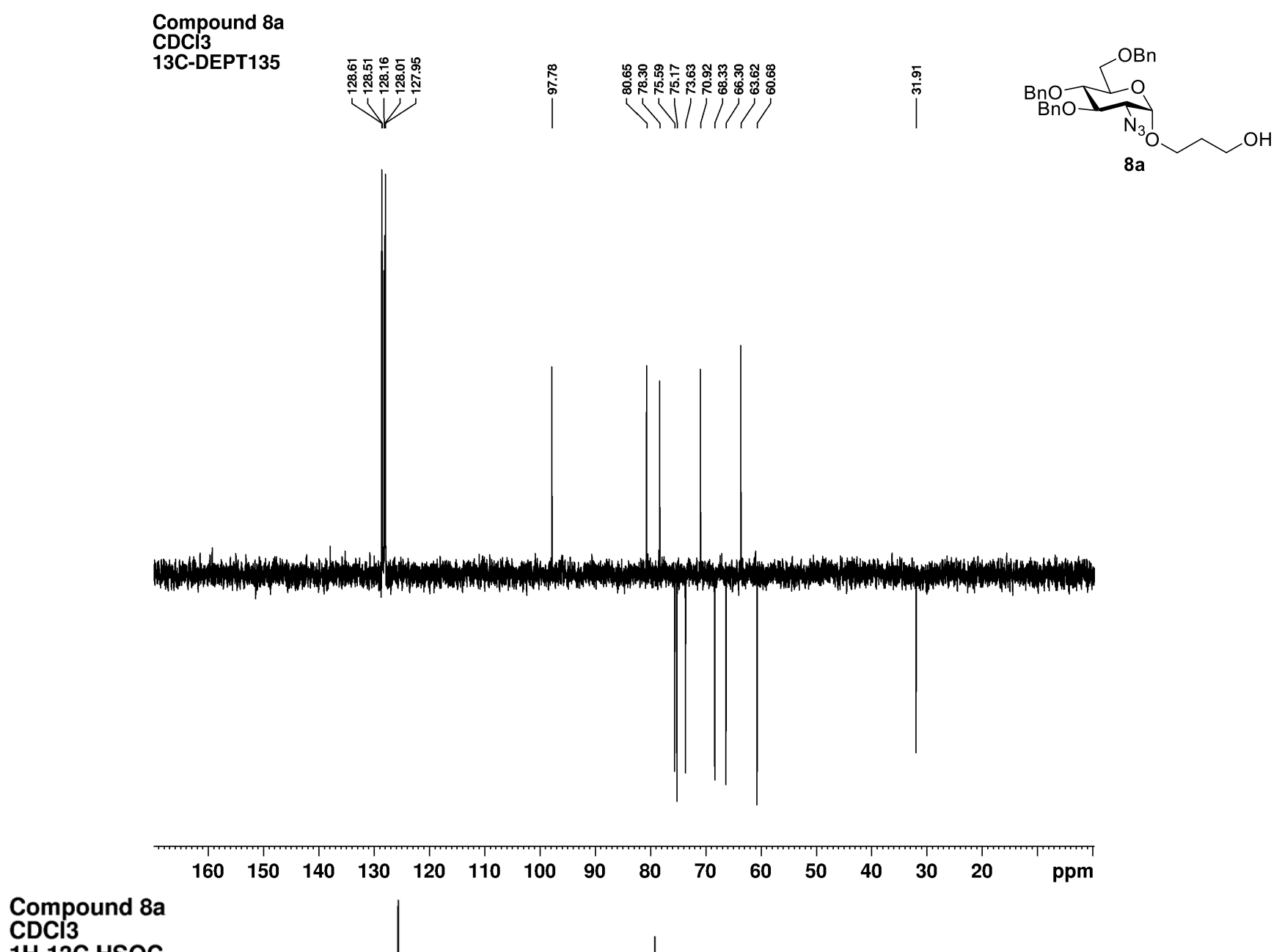

1H-13C HSQC

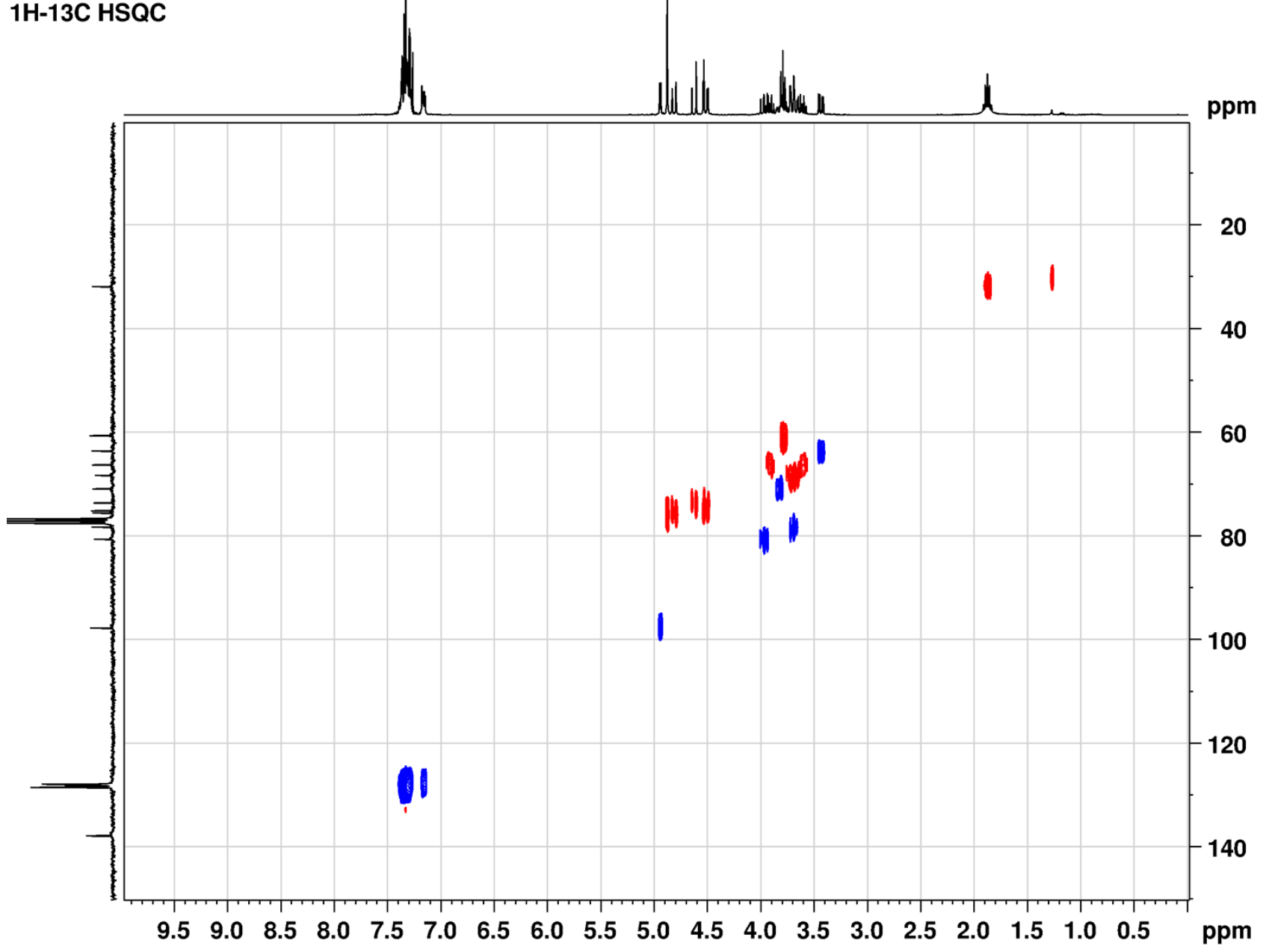


2.7. 2-hydroxypropyl 2-azido-3,4,6-tri-O-benzyl-2-deoxy- $\alpha$-D-glucopyranoside (8b)

${ }^{1} \mathrm{H} \mathrm{NMR}, \mathrm{CDCl}_{3}, 300 \mathrm{MHz}$

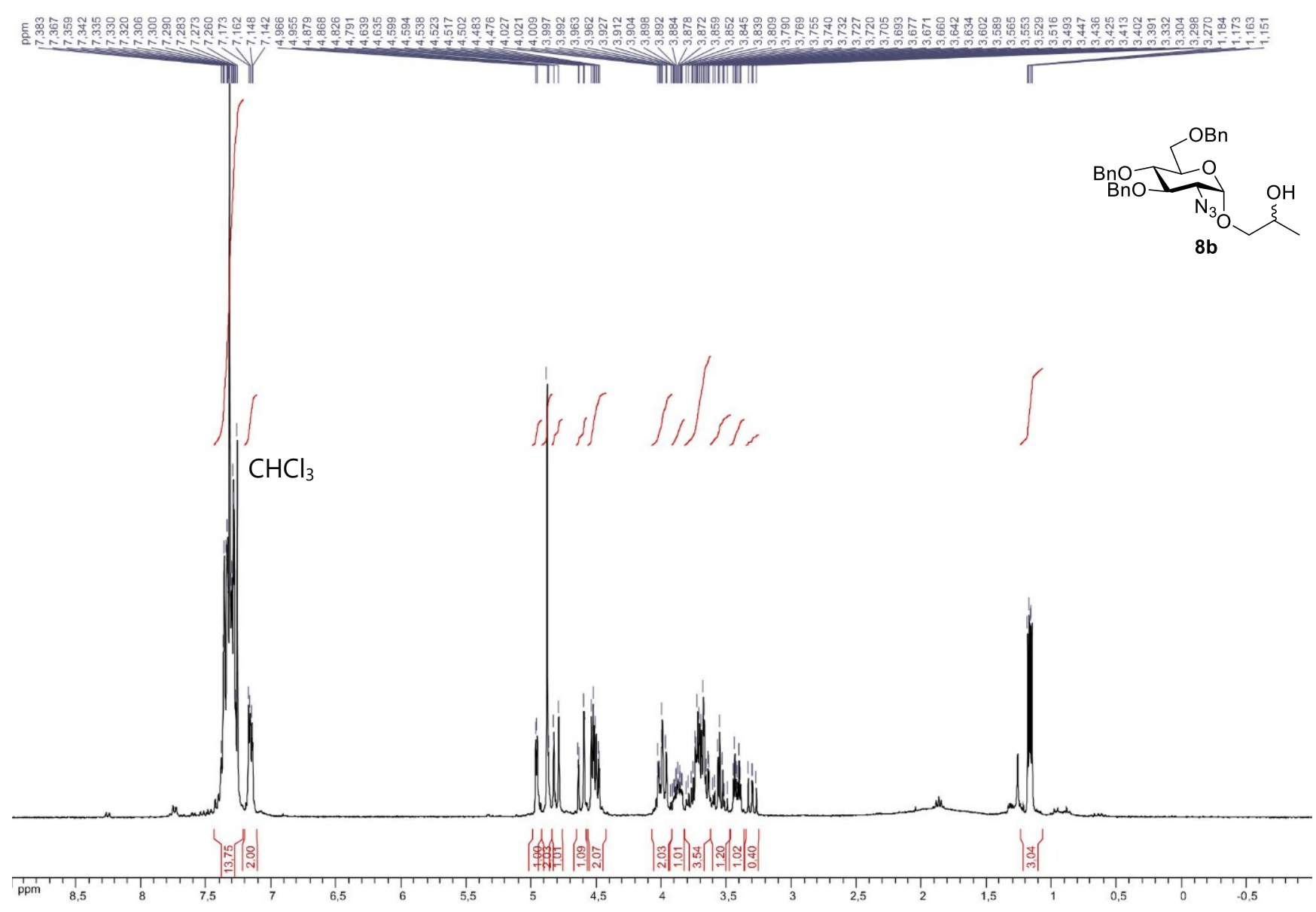

${ }^{13} \mathrm{C}-\left\{{ }^{1} \mathrm{H}\right\} \mathrm{NMR}, \mathrm{CDCl}_{3}, 75 \mathrm{MHz}$
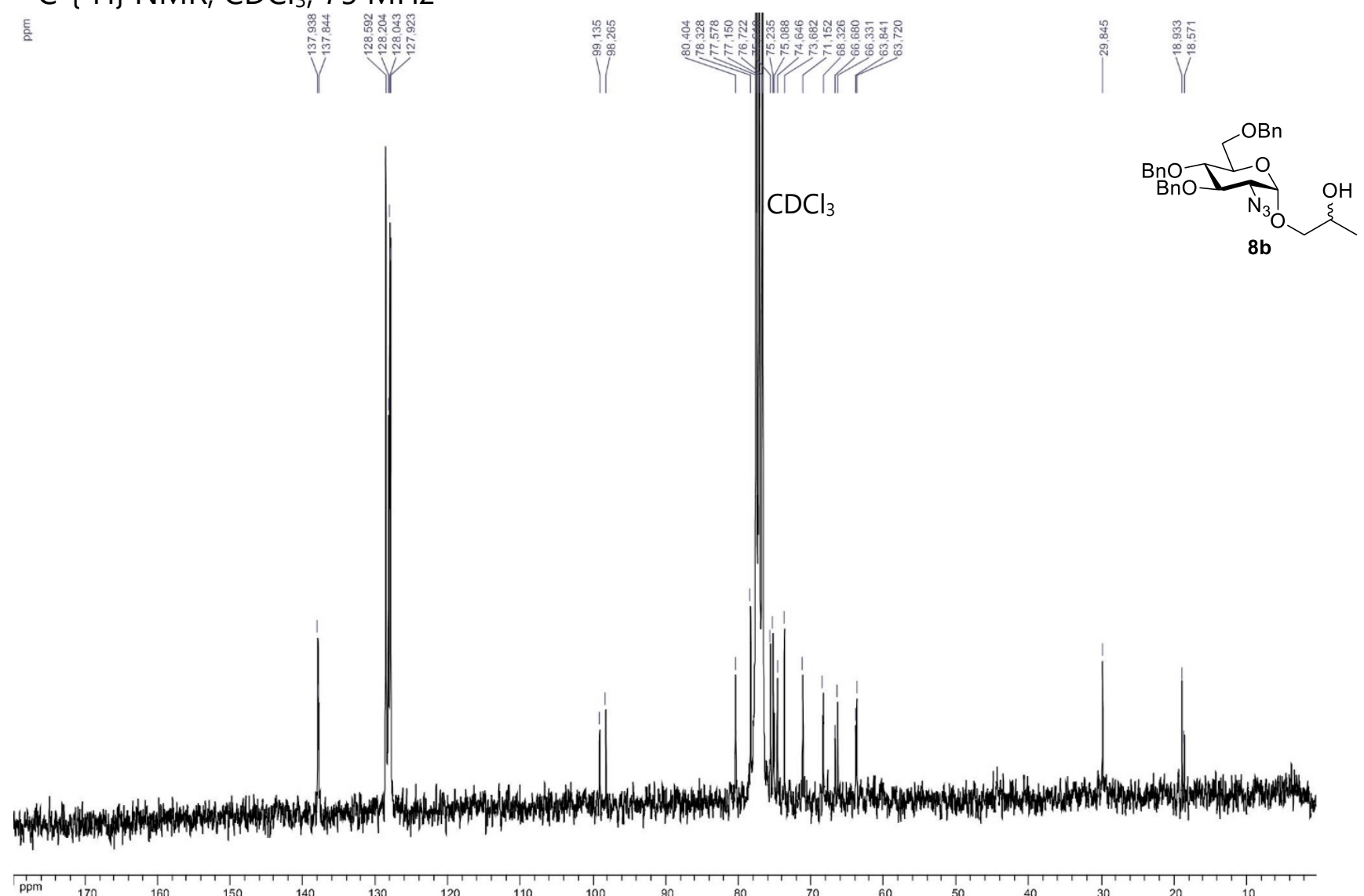


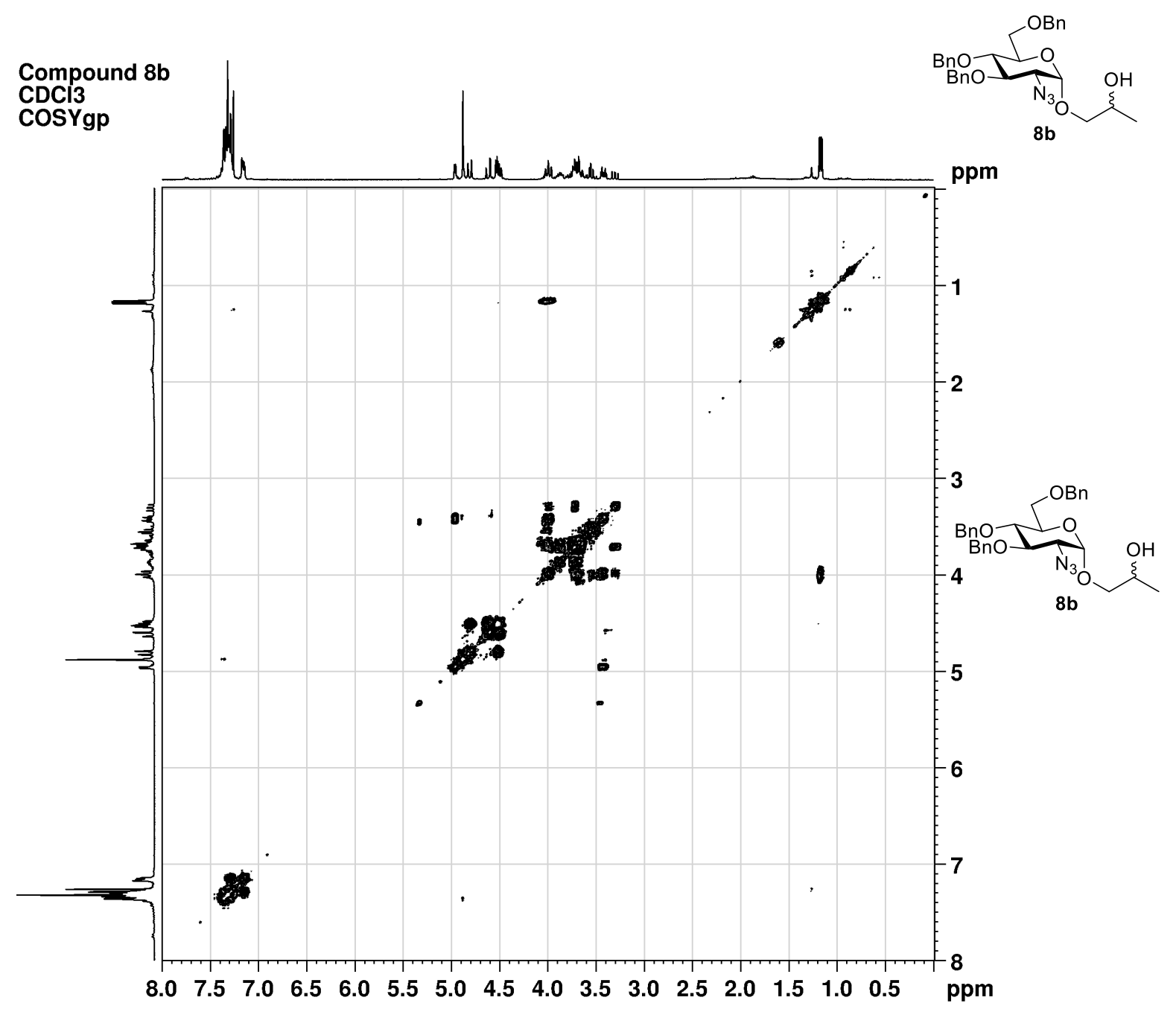

Compound 8b CosYg

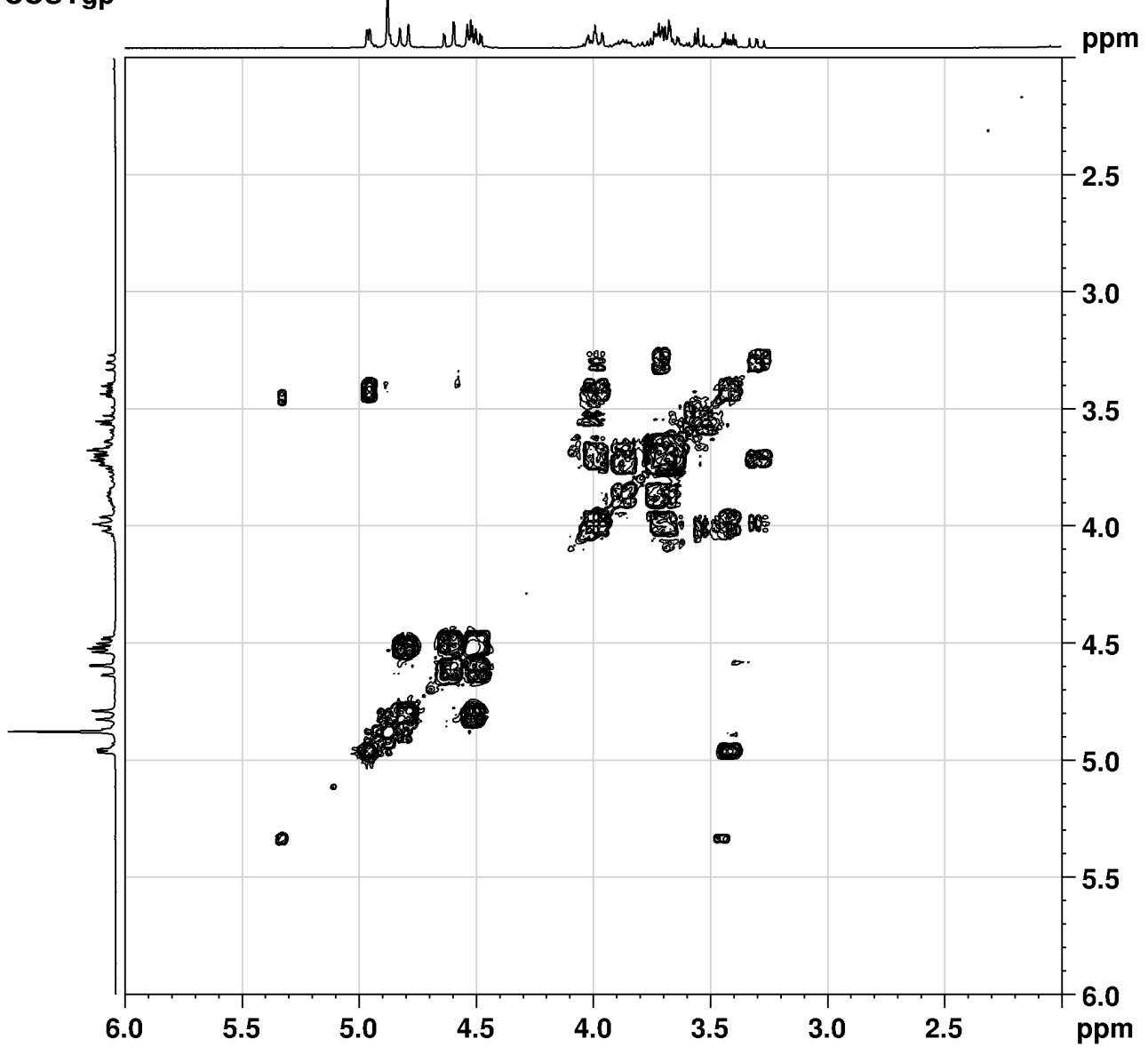



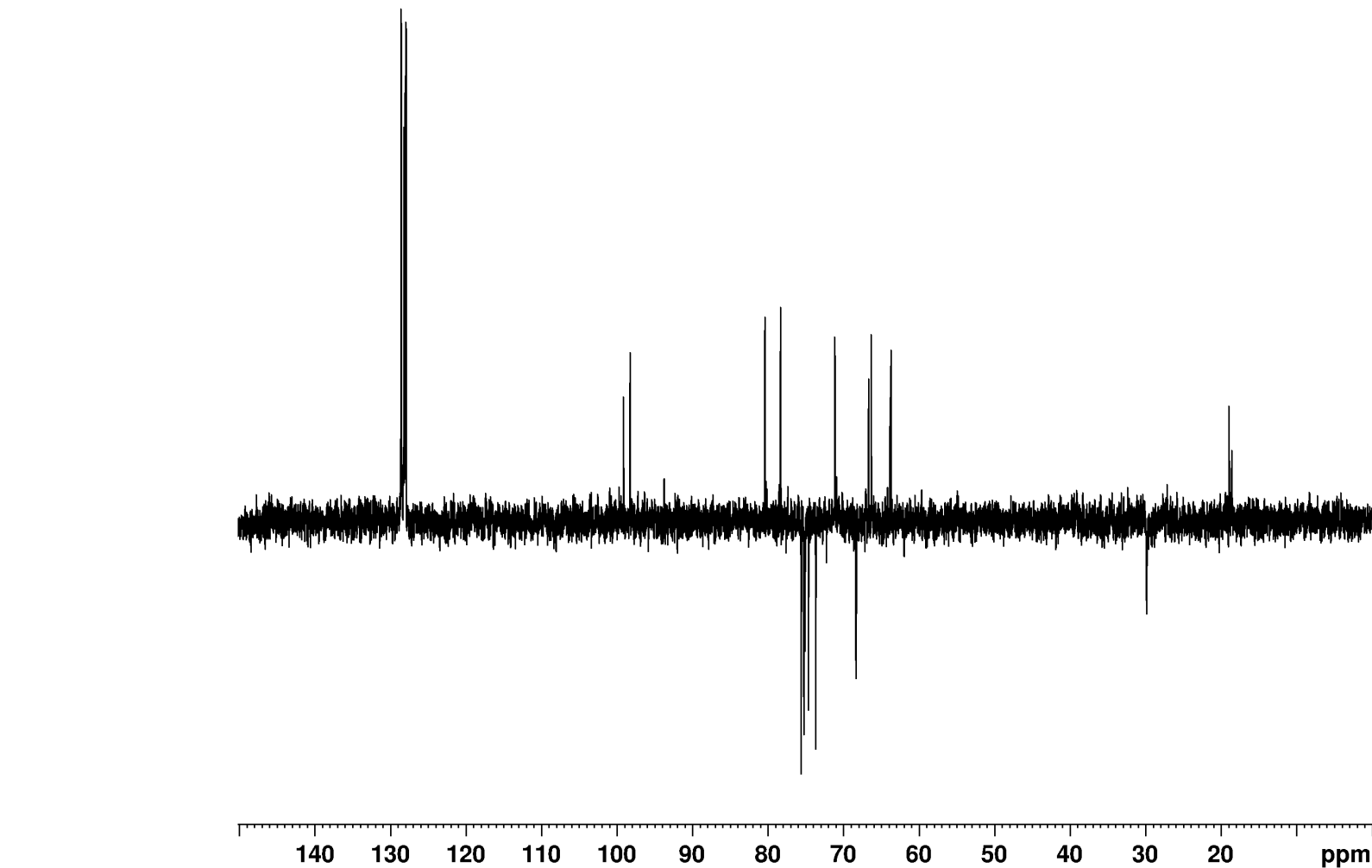

\section{Compound $8 \mathrm{~b}$} $\mathrm{CDCl} 3$

1H-13C HSQC
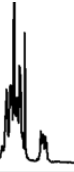

$\begin{array}{llll}130 & 120 & 110 & 100\end{array}$
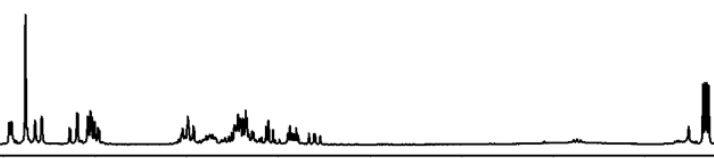

ppm

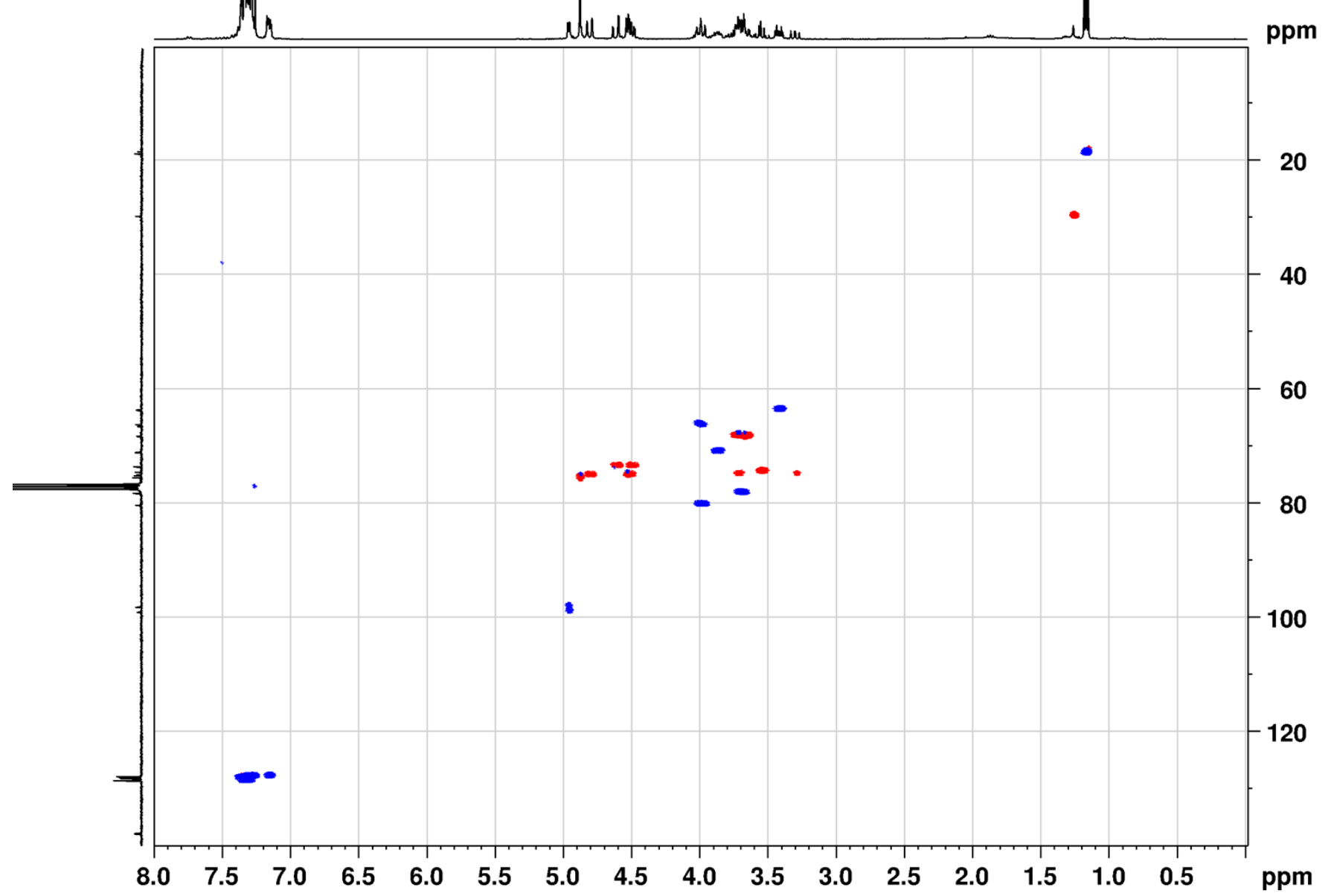




\section{HRMS data for compound 9}

\section{Mass Spectrum SmartFormula Report}

Analysis Info

Method

Sample Name

ms_fia_tw_50_1200_pos-calibrant1.8.m

Comment

Acquisition Date 01/08/2019 15:21:45

Operator BDAL@DE

Instrument / Ser\# micrOTOF-Q II 8228888.1

0027

\section{Acquisition Parameter}

Source Type

Focus

ESI

Scan Begin

Not active

$50 \mathrm{~m} / \mathrm{z}$

$1200 \mathrm{~m} / \mathrm{z}$

Ion Polarity

Set Capillary

Set End Plate Offset

Set Collision Cell RF

\section{Positive}

$4500 \mathrm{~V}$

$-500 \mathrm{~V}$

$50.0 \mathrm{Vpp}$

Set Dry Heater $\quad 180^{\circ} \mathrm{C}$

Set Dry Gas $\quad 9.0 \mathrm{l} / \mathrm{min}$

Set Divert Valve

Waste
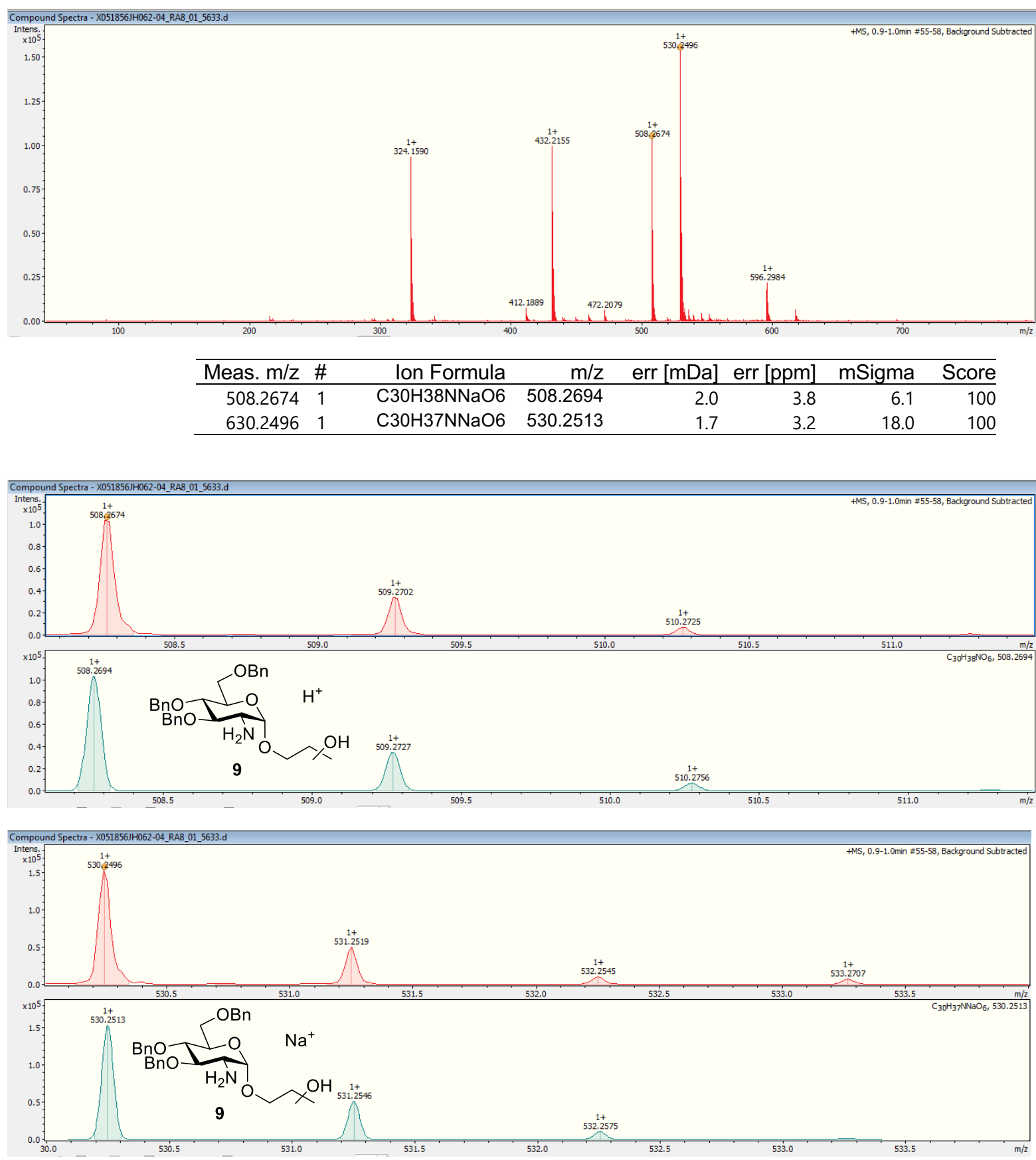
$4{ }^{1} \mathrm{H},{ }^{13} \mathrm{C}-\left\{{ }^{1} \mathrm{H}\right\}$, DEPT-135, COSY, ${ }^{1} \mathrm{H}-{ }^{13} \mathrm{C}$ HSQC and HRMS data for compound 10

${ }^{1} \mathrm{H} \mathrm{NMR}, \mathrm{CDCl}_{3}, 300 \mathrm{MHz}$

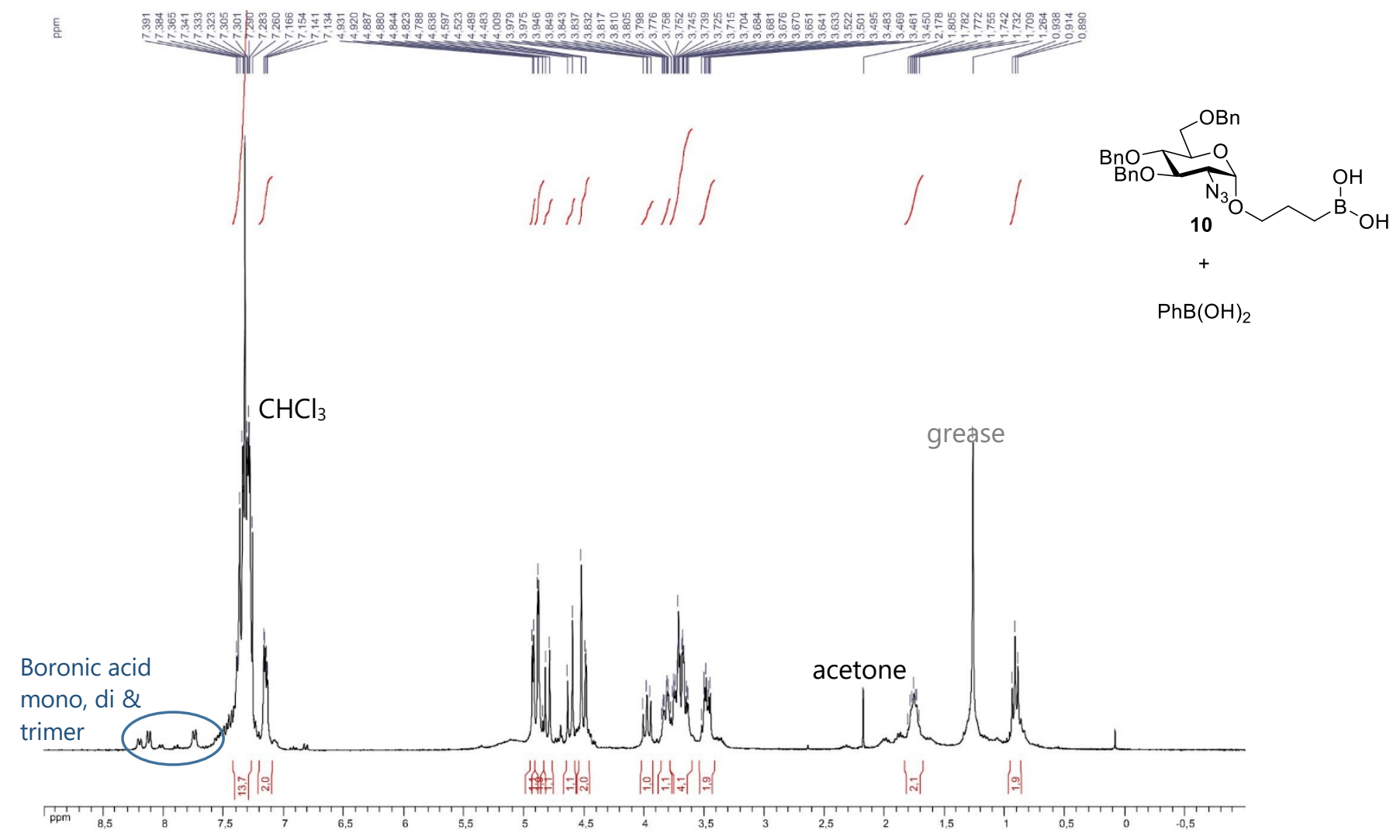

COSY NMR, $\mathrm{CDCl}_{3}, 300 \mathrm{MHz}$

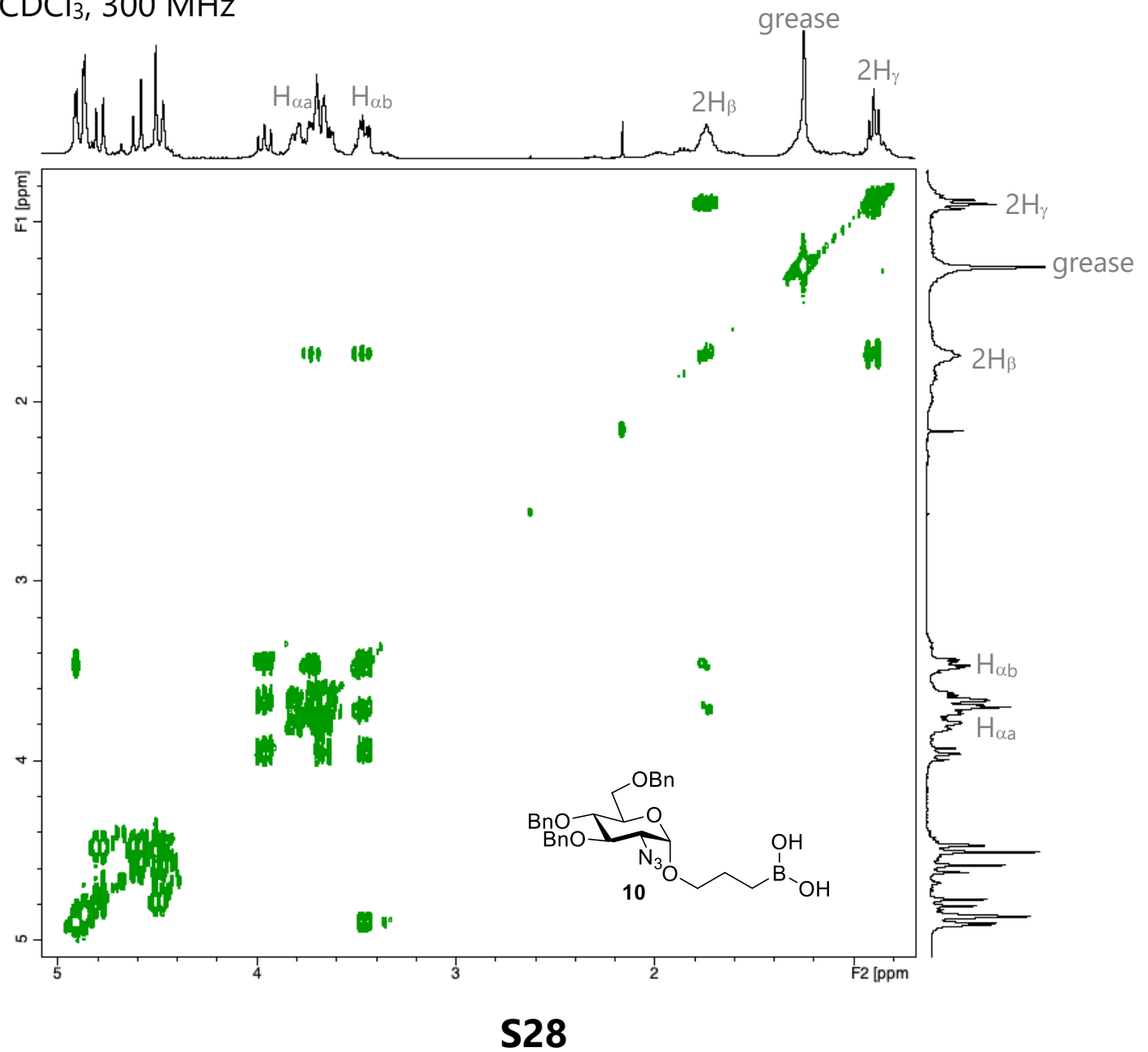


${ }^{13} \mathrm{C}-\left\{{ }^{1} \mathrm{H}\right\} \mathrm{NMR}$ and DEPT-135, CDCl $3,75 \mathrm{MHz}(\mathrm{AQ}=2.2 \mathrm{~s}, \mathrm{D} 1=2.0 \mathrm{~s}, \mathrm{NS}=1024)$

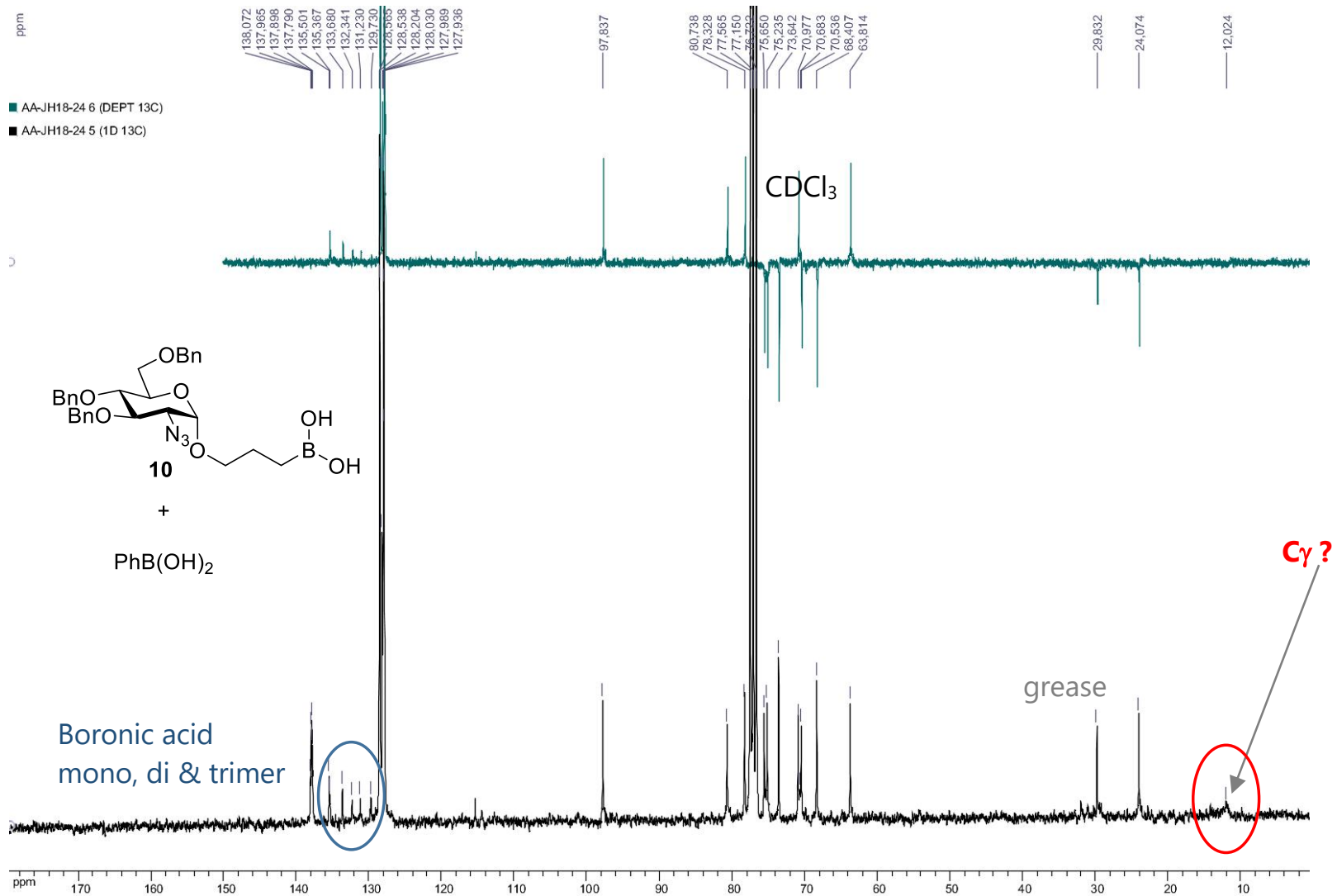

Short $\mathrm{T} 2$ for ${ }^{13} \mathrm{C} \gamma$ allowed shortening the acquisition times and recycling delays to increase the number of scan for ${ }^{13} \mathrm{C}-\left\{{ }^{1} \mathrm{H}\right\}$ : NMR, $\mathrm{CDCl}_{3}, 100 \mathrm{MHz}(\mathrm{AQ}=0.4 \mathrm{~s}, \mathrm{D} 1=0.5 \mathrm{~s}, \mathrm{NS}=204800)$

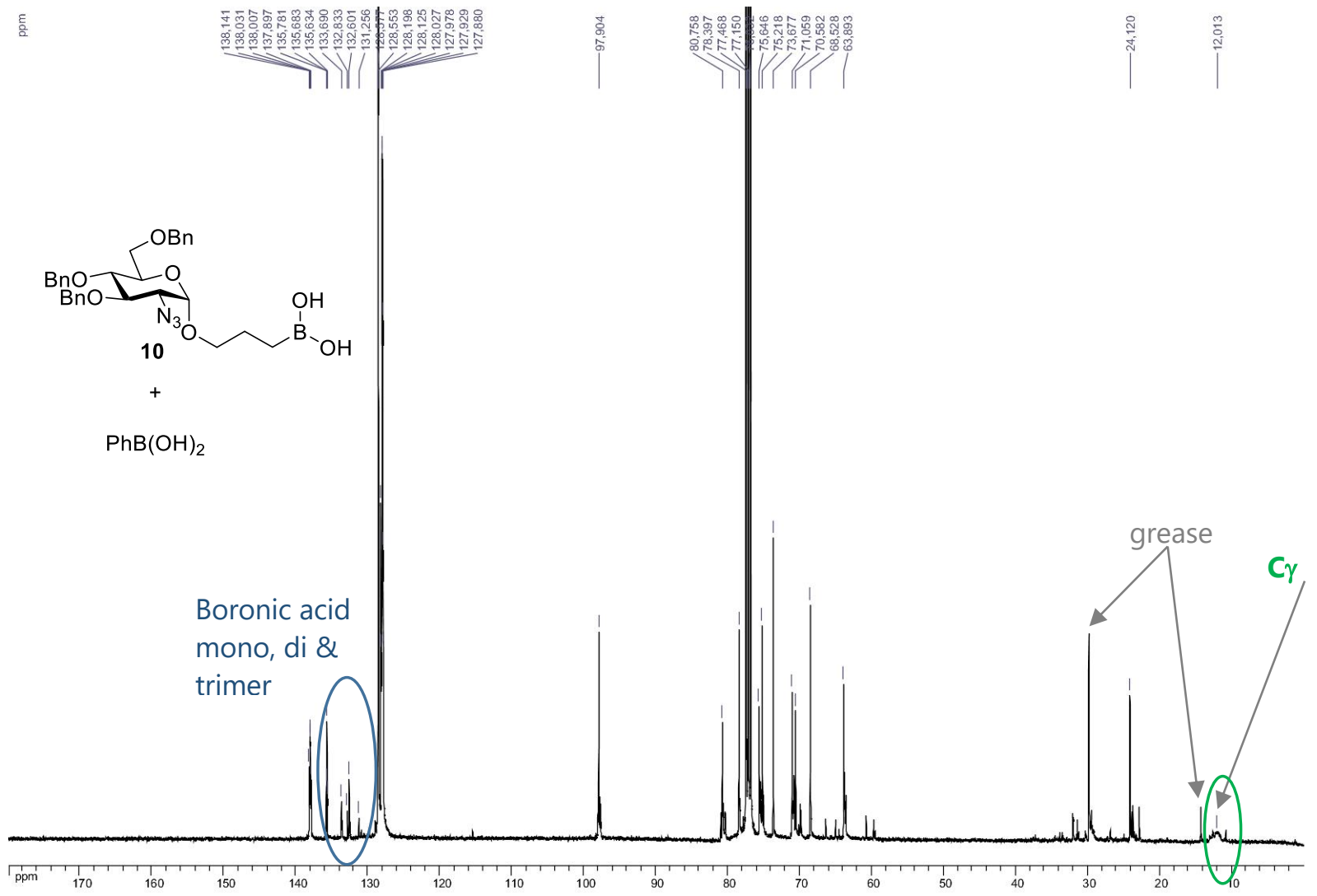


${ }^{1} \mathrm{H}^{-13} \mathrm{C}$ HSQC NMR, $\mathrm{CDCl}_{3}, 400 \mathrm{MHz}, \mathrm{F} 2: \mathrm{TD}=4096, \mathrm{AQ}=0.5 \mathrm{~s}(\mathrm{~F} 1) ; \mathrm{F} 1: \mathrm{TD}=512,0.016 \mathrm{~s} ; \mathrm{NS}=$ 16)

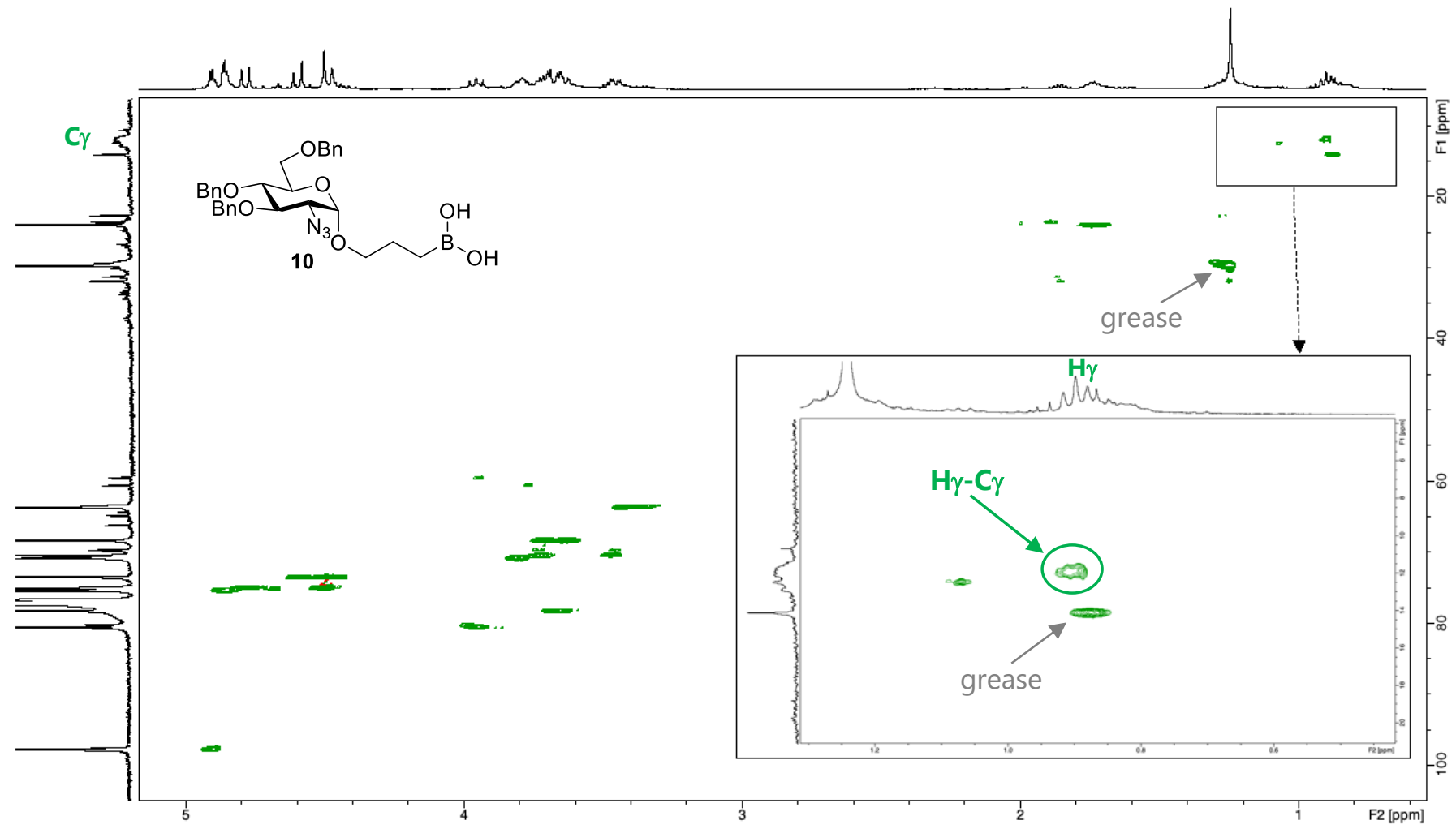

${ }^{1} \mathrm{H}-{ }^{13} \mathrm{C} \mathrm{HMBC} \mathrm{NMR}, \mathrm{CDCl}_{3}, 400 \mathrm{MHz}$

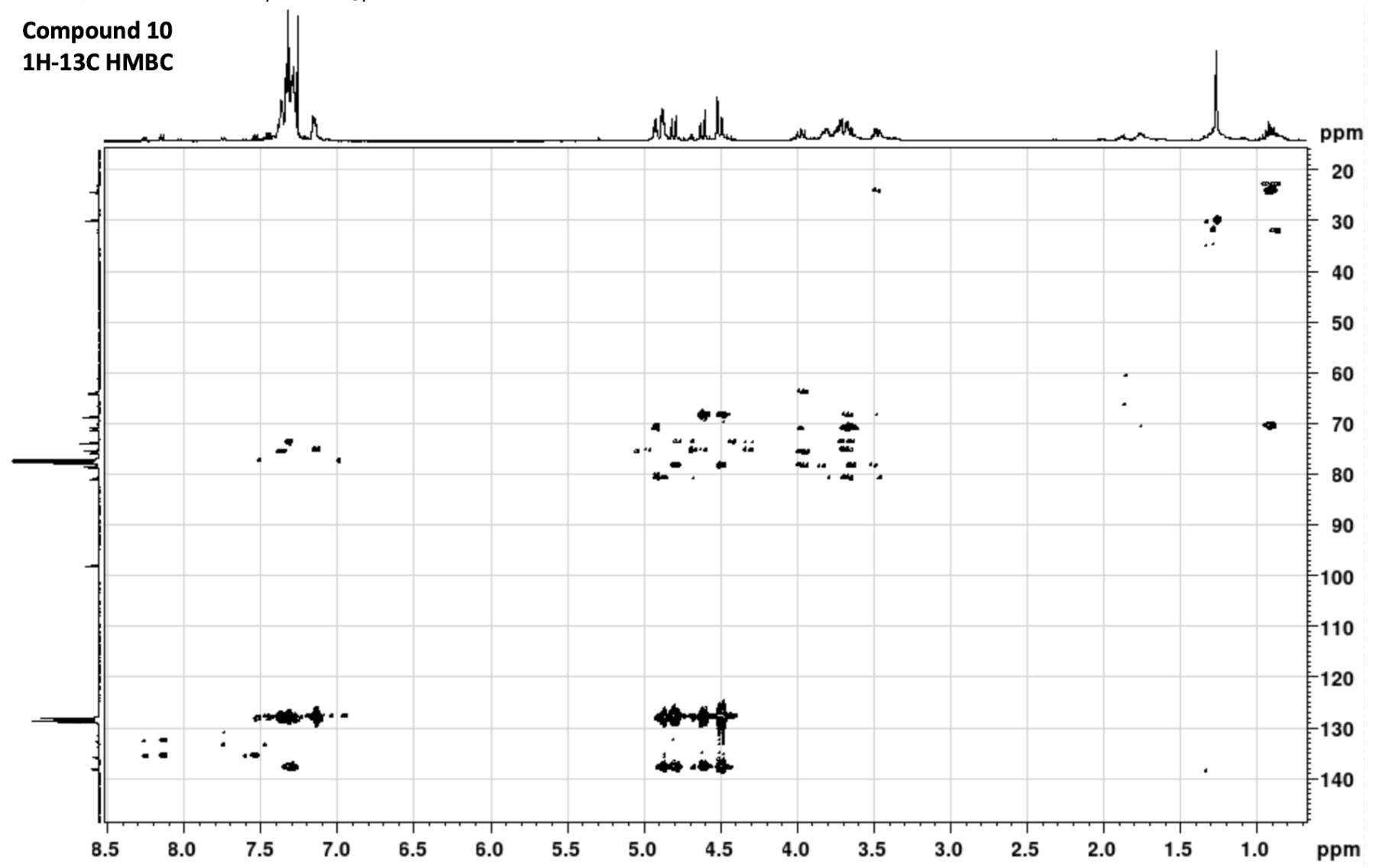


${ }^{11} \mathrm{~B} N M R, \mathrm{CDCl}_{3}, 96 \mathrm{MHz}$ : Compound 10 (black) and $\mathrm{PhB}(\mathrm{OH})_{2}$ as reference (blue)

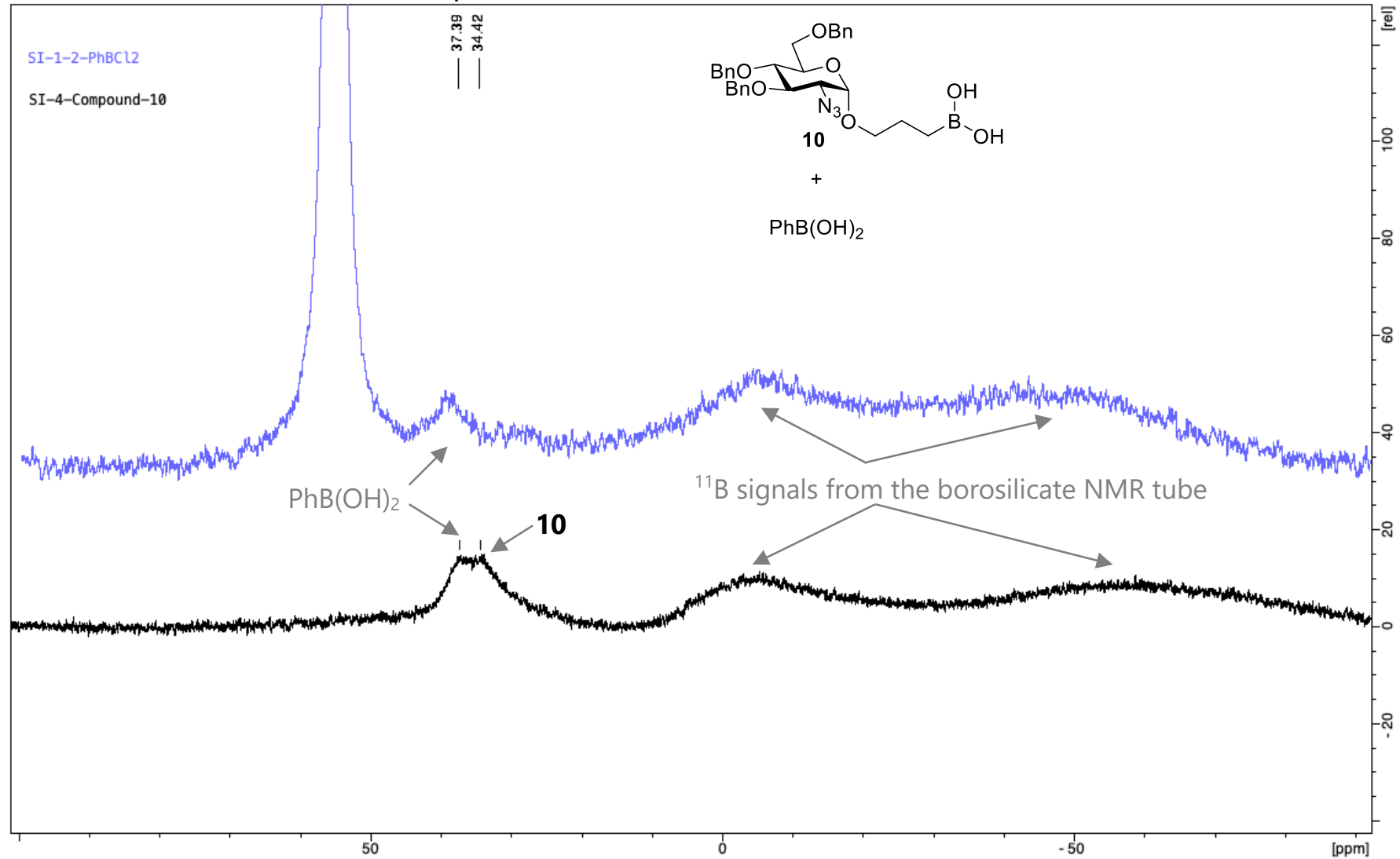


ESI HRMS of compound $\mathbf{1 0}$ (solution in methanol): identification of mono and dimethyl boronic ester derivatives

\section{Compound Spectrum SmartFormula Report}

\section{Analysis Info}

Analysis Name

Method

Sample Name

Comment
Acquisition Date 8/2/2019 2:54:35 PM

C:IUsers $\mid E C I D o c u m e n t s \backslash M a s s e l X 051856 J H 062-03 b i s-t w . d$

MS_Inf_TW_50_1200_pos.m

Operator BDAL@DE

Instrument micrOTOF-Q I| 8228888.10027

\section{Acquisition Parameter}

Source Type

Focus

Focus

Not active

Scan Begin

$100 \mathrm{~m} / \mathrm{z}$

$1200 \mathrm{~m} / \mathrm{z}$

$\begin{array}{ll}\text { Ion Polarity } & \text { Positive } \\ \text { Set Capillary } & 4500 \mathrm{~V} \\ \text { Set End Plate Offset } & -500 \mathrm{~V} \\ \text { Set Collision Cell RF } & 50.0 \mathrm{Vpp}\end{array}$

$\begin{array}{ll}\text { Set Nebulizer } & 0.4 \mathrm{Bar} \\ \text { Set Dry Heater } & 180^{\circ} \mathrm{C} \\ \text { Set Dry Gas } & 4.0 \mathrm{I} / \mathrm{min}\end{array}$

Set Divert Valve Waste

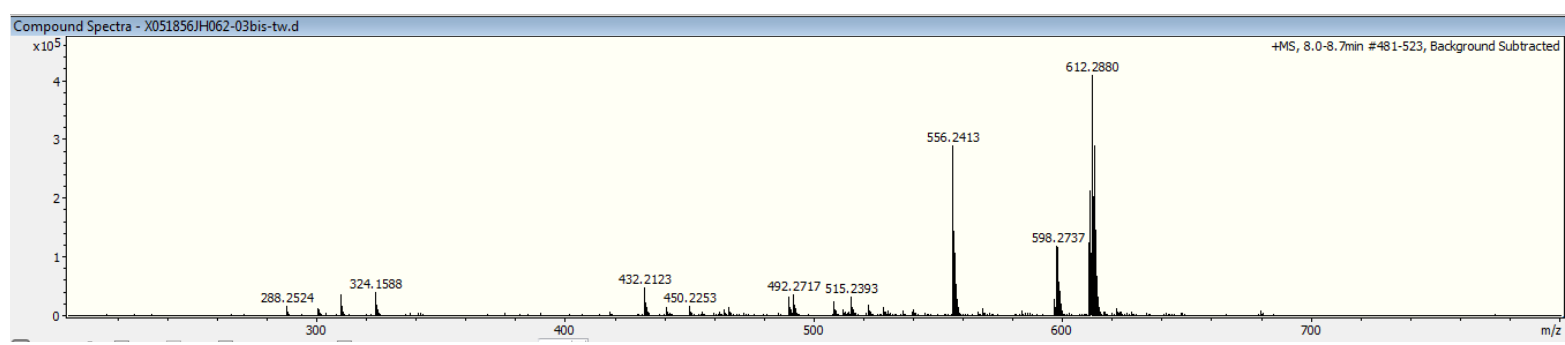

\begin{tabular}{|c|c|c|c|c|c|c|c|}
\hline Meas. $\mathrm{m} / \mathrm{z}$ & \# & Ion Formula & $\mathrm{m} / \mathrm{z}$ & err [mDa] & err [ppm] & mSigma & Score \\
\hline 598.2737 & 1 & C31H38BN3NaO7 & 598.2701 & -3.6 & -6.0 & 9.0 & 100 \\
\hline 612.2880 & 1 & $\mathrm{C} 32 \mathrm{H} 40 \mathrm{BN} 3 \mathrm{NaO} 7$ & 612.2857 & -2.3 & -3.8 & 222.6 & 100 \\
\hline
\end{tabular}
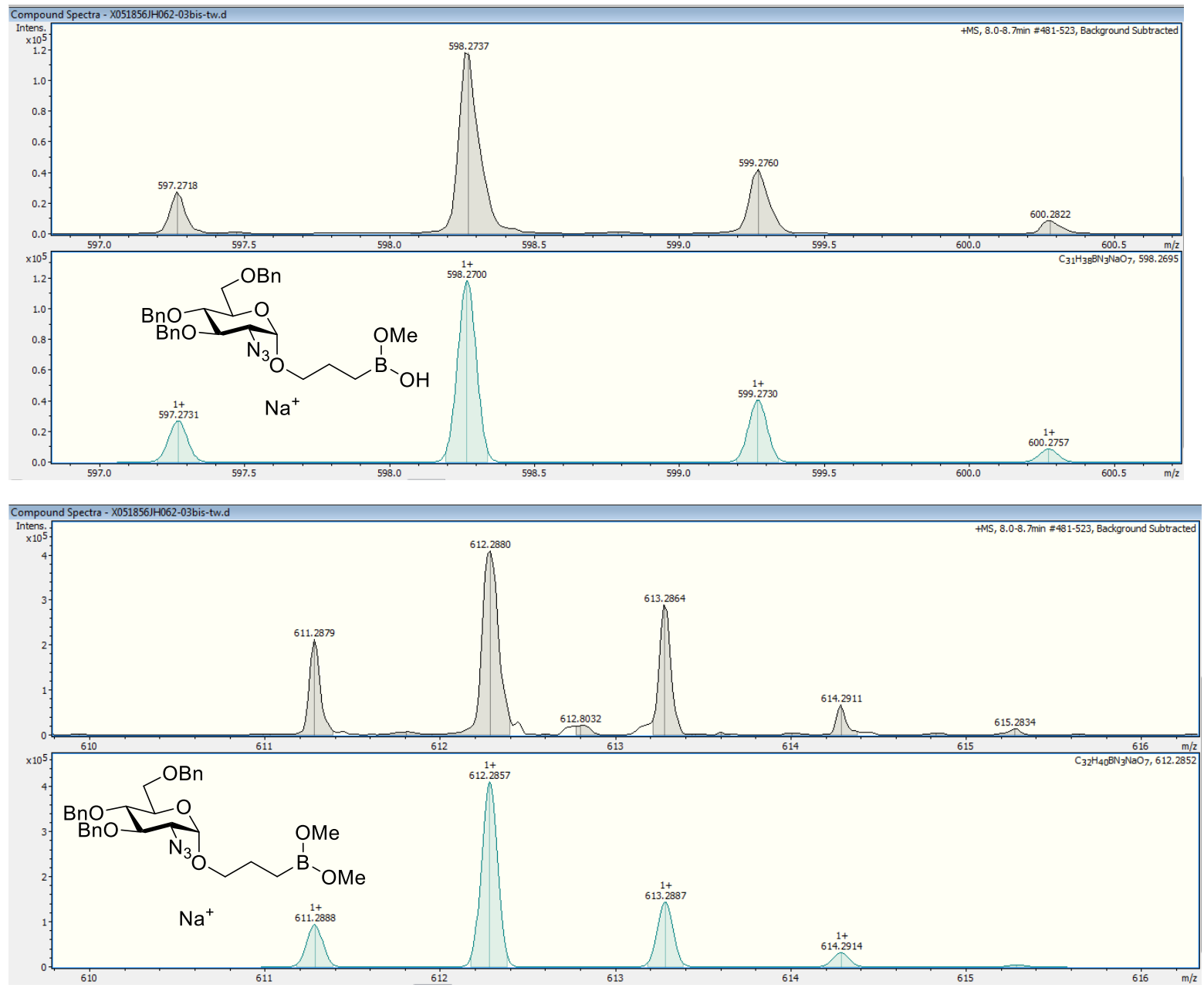

References:

1 Chaudhari, S. R. Screening and assignment of phenylboronic acid and its anhydride formation by NMR spectroscopy Chem. Phys. Lett. 2015, 634, 95-97. 